\title{
Neuroimaging and neuromodulation: complementary approaches for identifying the neuronal correlates of tinnitus
}

\section{Berthold Langguth ${ }^{1,2}$, Martin Schecklmann ${ }^{1,2}$, Astrid Lehner ${ }^{1,2}$, Michael Landgrebe ${ }^{1,2}$, Timm Benjamin Poeppl ${ }^{1,2}$, Peter Michal Kreuzer ${ }^{1,2}$, Winfried Schlee ${ }^{3}$, Nathan Weisz ${ }^{4}$, Sven Vanneste ${ }^{5}$ and Dirk De Ridder ${ }^{5}$}

${ }^{1}$ Department of Psychiatry and Psychotherapy, University of Regensburg, Regensburg, Germany

${ }^{2}$ Tinnitus Center, University of Regensburg, Regensburg, Germany

${ }^{3}$ OBOB Laboratory, Department of Psychology, University of Konstanz, Konstanz, Germany and University of Trento, Trento, Italy

${ }^{4}$ Clinical and Biological Psychology, Institute of Psychology and Education, University of Ulm, Ulm, Germany

${ }^{5}$ TRI, BRAR ${ }^{2}$ and Department of Neurosurgery, University Hospital Antwerp, Antwerp, Belgium

\section{Edited by:}

Jos J. Eggermont, University of

Calgary, Canada

Reviewed by:

Jos J. Eggermont, University of Calgary, Canada

Steven W. Cheung, University of California San Francisco, USA

*Correspondence:

Berthold Langguth, Department of Psychiatry and Psychosomatics, Bezirksklinikum, University of Regensburg, Universitaetsstr. 84, 93053 Regensburg, Germany. e-mail: berthold.langguth@medbo.de
An inherent limitation of functional imaging studies is their correlational approach. More information about critical contributions of specific brain regions can be gained by focal transient perturbation of neural activity in specific regions with non-invasive focal brain stimulation methods. Functional imaging studies have revealed that tinnitus is related to alterations in neuronal activity of central auditory pathways. Modulation of neuronal activity in auditory cortical areas by repetitive transcranial magnetic stimulation (rTMS) can reduce tinnitus loudness and, if applied repeatedly, exerts therapeutic effects, confirming the relevance of auditory cortex activation for tinnitus generation and persistence. Measurements of oscillatory brain activity before and after rTMS demonstrate that the same stimulation protocol has different effects on brain activity in different patients, presumably related to interindividual differences in baseline activity in the clinically heterogeneous study cohort. In addition to alterations in auditory pathways, imaging techniques also indicate the involvement of non-auditory brain areas, such as the fronto-parietal "awareness" network and the non-tinnitus-specific distress network consisting of the anterior cingulate cortex, anterior insula, and amygdale. Involvement of the hippocampus and the parahippocampal region putatively reflects the relevance of memory mechanisms in the persistence of the phantom percept and the associated distress. Preliminary studies targeting the dorsolateral prefrontal cortex, the dorsal anterior cingulate cortex, and the parietal cortex with rTMS and with transcranial direct current stimulation confirm the relevance of the mentioned non-auditory networks. Available data indicate the important value added by brain stimulation as a complementary approach to neuroimaging for identifying the neuronal correlates of the various clinical aspects of tinnitus.

Keywords: chronic tinnitus, neuromodulation, neuroimaging, neuronal correlates, brain stimulation

\section{INTRODUCTION}

Tinnitus is a common and distressing symptom that is characterized by the perceived sensation of sound in the absence of an external stimulus, most commonly known as the perception of "ringing in the ears"). Moreover it has been generally recognized that tinnitus is clinically heterogeneous, with respect to its etiology, its perceptual characteristics and its accompanying symptoms. Neuroimaging studies have increasingly contributed to a better understanding of the neuronal correlates of the different forms of tinnitus. In detail they demonstrated involvement of both auditory pathways and non-auditory brain areas, such as dorsolateral prefrontal cortex, orbitofrontal cortex, anterior cingulate, subgenual cingulate, posterior cingulate, parietal cortex, amygdala, hippocampus, parahippocampus, and cerebellum (for review see Lanting et al., 2009). In a recent working model these areas were suggested to be implicated in attentional, emotional, cognitive, and memory aspects related to tinnitus (De Ridder et al., 2011a). However an inherent limitation of functional imaging studies is its correlational approach. In other words, functional imaging can only reveal alterations of neuronal activity that are related to tinnitus, but cannot distinguish, which alterations are of causal relevance and which may just represent epiphenomena. More information about critical contributions of specific brain regions can be gained by transient perturbation of neural activity in these regions. This can be done by investigating the behavioral effects of focal brain stimulation methods. Brain stimulation techniques can be non-invasive, e.g., transcranial magnetic stimulation (TMS) or transcranial direct current stimulation (tDCS) or invasive, e.g., epidural or deep brain electrical stimulation.

Notably the still incomplete understanding of the mechanisms by which brain stimulation methods exert their behavioral effects limits their informative value for mapping brain function 
(Borchers et al., 2011). TMS for example represents a multimodal stimulation approach involving stimulation of somatosensory afferents and auditory stimulation in addition to cortical stimulation, all of which may be relevant for the observed behavioral effect (Schecklmann et al., 2011b; Vanneste et al., 2011b; Zunhammer et al., 2011). However, with these limitations in mind brain stimulation techniques still represent useful tools for complementing neuroimaging techniques in the study of brain function by testing hypotheses of causal relationships between the behavioral effects and imaging results. In detail, performing neuroimaging before and after brain stimulation can directly reveal, which changes of brain activity are related to subjective perceptual changes. Here we will review how neuroimaging and brain stimulation studies have complemented each other in the identification of neuronal correlates of tinnitus.

\section{BRAIN STIMULATION TECHNIOUES TRANSCRANIAL MAGNETIC STIMULATION}

Transcranial magnetic stimulation is an experimental tool for stimulating neuronal cell assemblies via brief magnetic pulses delivered by a coil placed on the scalp (Barker et al., 1985). A short lasting, high intensity current pulse through an insulated stimulating coil induces a magnetic field perpendicular to the coil which penetrates the scalp with little attenuation inducing an electrical current in the brain area under the coil, which in turn induces depolarization of nerve cells. Magnetic coils with a variety of shapes are available. Figure-eight-shaped coils are preferentially used, since they produce a more focal magnetic field than round coils. Their maximal current is delivered at the intersection of the two round components (Hallett, 2000). Due to the strong decline of the magnetic field with increasing distance from the coil, the direct stimulation is limited to superficial cortical areas. However, stimulation effects propagate transsynaptically to functionally connected remote areas and thus modulate brain network activity (Siebner et al., 2003; May et al., 2007). More recently newer coils have been developed that might be able to penetrate deeper into the brain, such as the $\mathrm{H}$-coil (Rosenberg et al., 2011) or double cone coil (Hayward et al., 2007).

Whereas single magnetic pulses do not seem to have longer lasting effects on the brain, the application of multiple pulses, called repetitive TMS (rTMS), can induce changes in neuronal excitability that outlast the duration of the stimulation (Hallett, 2000). These effects resemble those seen in animal experiments where repeated electrical stimulation has been shown to produce changes in the effectiveness of synapses in the same circuits (Hoffman and Cavus, 2002). These changes include the phenomena of long term potentiation (LTP) and long term depression (LTD), which have been shown to be important for learning and memory processes (Wang et al., 1996). rTMS can also be used to transiently disturb ongoing neural activity in the stimulated cortical area, thus creating a transient functional lesion. Such an approach can help to identify whether a given brain area is critically involved in a specific behavioral task. However in the interpretation of the effects it has to be considered that effects of rTMS are not limited to the directly stimulated brain regions, but can also induce changes in remote functionally connected brain areas. Moreover it has to be taken into consideration that rTMS always represents a multimodal stimulation approach involving not only cortical stimulation, but also the stimulation of somatosensory afferents (Vanneste et al., 2011b; Zunhammer et al., 2011) by the stimulation of scalp neurons and auditory stimulation (Schecklmann et al., 2011b) by its acoustic artifact, all of which may be relevant for the observed behavioral effect.

\section{TRANSCRANIAL DIRECT CURRENT STIMULATION}

Transcranial Direct Current stimulation is another non-invasive procedure for cortical stimulation. For tDCS a relatively weak constant direct current (between 0.5 and $2 \mathrm{~mA}$ ) is transiently applied via scalp electrodes. The current flows from the anode to the cathode (George and ston-Jones, 2010), and about 50\% of the current is shunted through the skin and subcutaneous tissues, whereas $50 \%$ goes through the brain (Dymond et al., 1975). Depending on the polarity of the stimulation, $\mathrm{tDCS}$ can increase or decrease cortical excitability in the brain regions under the electrode (Nitsche and Paulus, 2000). Anodal tDCS typically has an excitatory effect on the local cerebral cortex by depolarizing neurons, while the opposite is the case under the cathode, where hyperpolarization occurs. This effect of tDCS typically outlasts the stimulation by an hour or longer after a single treatment session of about 20-30 min (Nitsche and Paulus, 2000, 2001)

\section{EPIDURAL STIMULATION}

Epidural stimulation via implanted electrodes is an invasive neuromodulation technique used to permanently modulate activity of the cerebral cortex, which is in contrast to non-invasive techniques that usually yield a transient modulation. Electrodes can be placed anywhere on the cortex, e.g., motor cortex (Nguyen et al., 1997), somatosensory cortex (De Ridder et al., 2007b), auditory cortex (De Ridder et al., 2007a), or dorsolateral prefrontal cortex (De Ridder et al., 2011d). The target is usually retrieved by functional imaging such as fMRI (De Ridder et al., 2004) or PET scan, but can also be done using a combination of intraoperative electrophysiological measures (ERP; Pirotte et al., 2005) and functional imaging data (MRI; Pirotte et al., 2008). Attempts have been made to use non-invasive TMS prognostically showing moderate (De Ridder et al., 2011c) to good results (Lefaucheur et al., 2011).

\section{DEEP BRAIN STIMULATION}

Deep brain stimulation (DBS) uses the same technique as epidural stimulation to modulate electrical activity in the deeper brain structures. It has been shown that DBS alters neurotransmitter release and electrical activity locally, and also exerts a certain network effect (Kringelbach et al., 2007). For localization purposes Cartesian coordinates derived from brain atlases are used to integrate in stereotactic frames. This permits to accurately positioning a wire electrode in the elected neurostimulation target. Recordings from the inserted electrode can be performed for confirming the localization of the electrode at the intended target.

\section{THE INVOLVEMENT OF CENTRAL AUDITORY PATHWAYS IN TINNITUS}

Traditionally, tinnitus was considered to be a disorder that was primarily confined to the ear. However, the observation that tinnitus persists in general after transsection of the auditory nerve clearly 
indicates its central nervous system origin (House and Brackmann, 1981). Both animal models of tinnitus and neuroimaging research in patients suffering from tinnitus have provided important insight into the neuronal mechanisms involved in the pathophysiology of tinnitus. Based on animal studies, three mechanisms have been proposed to underlie tinnitus: (1) changes in the firing rates of spontaneous neural activity in the central auditory system, (2) changes in the temporal pattern of neural activity (synchrony), and (3) reorganization of tonotopic maps (Eggermont and Roberts, 2004).

The first functional neuroimaging studies of tinnitus have been focused on the auditory system either by analyzing steady-state neural activity in the auditory cortex (Arnold et al., 1996) or by investigating sound-evoked responses (Lockwood et al., 1998; Melcher et al., 2000). All studies with $\left[{ }^{15} \mathrm{O}\right]-\mathrm{H}_{2} \mathrm{O}$ PET have consistently provided evidence for tinnitus-related elevated blood flow in auditory structures. Measurements of regional glucose uptake (FDG-PET), which is a marker for steady-state neuronal activity, found an asymmetric activation of the auditory cortex with an increase predominantly on the left side and independent of tinnitus perceived laterality (Arnold et al., 1996; Langguth et al., 2006a). In several (Melcher et al., 2000; Smits et al., 2007; Lanting et al., 2008) but not all (Melcher et al., 2009) studies investigating individuals with unilateral tinnitus, altered activation patterns were observed in the auditory pathway contralateral to where the tinnitus was perceived.

Alterations of neuronal activity in central auditory pathways have also been investigated by electroencephalography (EEG) and magnetoencephalography (MEG). In people with chronic tinnitus MEG (Llinas et al., 1999; Weisz et al., 2005b, 2007) and EEG (van der Loo et al., 2009; Moazami-Goudarzi et al., 2010) resting state measurements revealed relatively consistent a reduction of alpha activity $(8-12 \mathrm{~Hz})$, and an increase in both slow wave activity (delta and theta $1-6 \mathrm{~Hz})$ and gamma activity $(>30 \mathrm{~Hz})$ in the temporal cortex.

Alterations of neuronal activity in auditory pathways of tinnitus patients have also been documented by using auditory evoked potentials, where both increases (Santos and Matas, 2010) and decreases (Attias et al., 1993, 1996) of amplitudes have been found. The increases were observed in patients without hearing loss (i.e., $<25 \mathrm{~dB}$; Santos and Matas, 2010), whereas the decreases in patients with hearing loss (Attias et al., 1993). Significantly increased N1P2 amplitudes were found at higher stimulus intensities for the tinnitus ear in comparison to the non-tinnitus ear in patients with unilateral tinnitus (Norena et al., 1999).

It is assumed that the observed alterations of neural activity in the auditory pathways arise as a consequence of altered sensory input, namely auditory deprivation in most cases (Norena et al., 2002; Norena and Eggermont, 2005), but can also be due to altered somatosensory input (Roberts et al., 2010). Several lines of evidence indicate that the mentioned adaptive processes are mainly driven by mechanisms of homeostatic plasticity which alter the balance between excitatory and inhibitory function of the auditory system at several levels in order to compensate for the reduced input (Schaette and Kempter, 2006; Norena, 2011; Schaette and McAlpine, 2011; Yang et al., 2011).
Even if neuroimaging findings largely contributed to a more detailed understanding of tinnitus, important knowledge gaps still remain. Thus it is not clear to which extent the observed findings are really related to tinnitus ("state") or whether they reflect a predisposition for developing tinnitus ("trait").

Moreover, the alterations of neuronal structure and function in tinnitus patients may represent the neuronal correlates of tinnitus, but they may also represent compensatory mechanisms for auditory deprivation and be unrelated to tinnitus or even beneficial for attenuating tinnitus. Answering these questions is challenging due to (1) the lack of longitudinal studies in humans with neuroimaging measurements before and after tinnitus onset, (2) the limited reliability of behavioral assessment of tinnitus in animals, and (3) the limited sensitivity for assessing auditory dysfunction in humans. Many cross-sectional imaging studies which compared tinnitus patients and controls, did not control for hearing loss. Thus it remains unclear whether the observed changes are related to tinnitus or to hearing loss. But even when the studies controlled for hearing loss, this was done based on the audiogram which provides only limited information about the integrity of the cochlea (Schaette and McAlpine, 2011).

Thus, the modulation of neuronal activity in the auditory pathways of tinnitus patients by means of focal brain stimulation represents an elegant way to further address the question whether alterations in the auditory pathway depict a trait or a state factor

\section{MODULATION OF AUDITORY CORTEX ACTIVITY Transcranial magnetic stimulation}

As mentioned before, tinnitus is related to altered activity of central auditory areas. If the perception of tinnitus can be influenced by rTMS over auditory cortical areas and given that rTMS reliably changes auditory cortex activity, this would provide support for a causal relationship between abnormal neural activity in these areas and tinnitus perception. Many studies have investigated the effects of both single sessions and repeated sessions of rTMS over temporal or temporoparietal brain areas.

Single sessions of rTMS for transient tinnitus suppression. Within the last years 11 studies involving over 300 patients have been published, in which single sessions of rTMS over temporal or temporoparietal areas have been applied (see Table 1). These studies differed with respect to the applied stimulation protocols, the exact stimulation areas, the method for coil localization, the chosen control condition, and the used assessment instruments. Nevertheless almost all studies reported a transient tinnitus reduction in a subgroup of tinnitus patients (for detailed results see Table 1). This indicates the relevance of the stimulated area for tinnitus perception in those patients. Only few studies have compared different stimulation protocols.

In one study different brain areas were stimulated with highfrequency rTMS $(10 \mathrm{~Hz})$. Best tinnitus suppression was found for stimulation of the left temporoparietal cortex resulting in a transient reduction of tinnitus in $57 \%$ of the participants (Plewnia et al., 2003).

In one study rTMS at frequencies between 1 and $20 \mathrm{~Hz}$ was applied over the auditory cortex contralateral to the site of tinnitus 


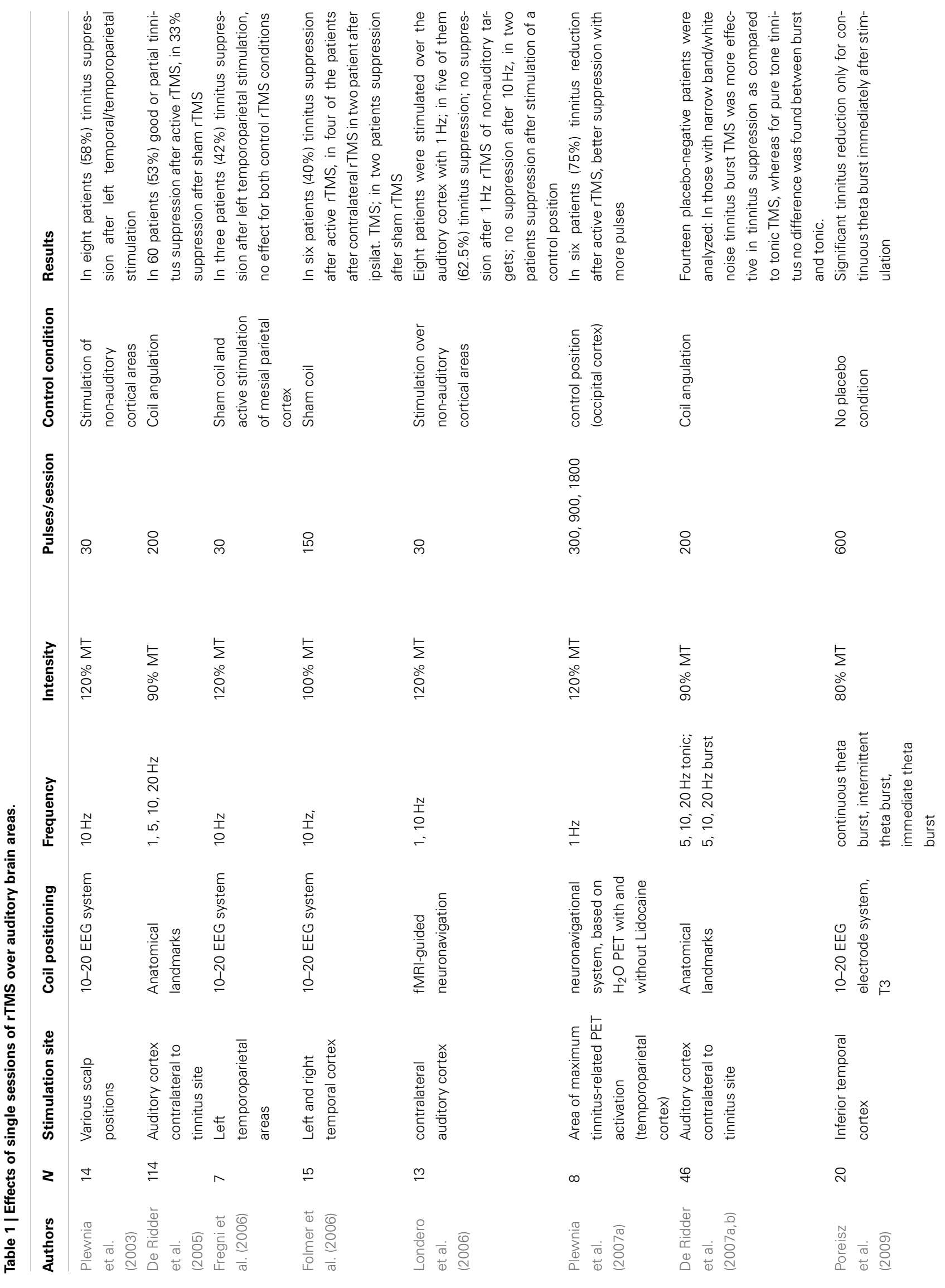



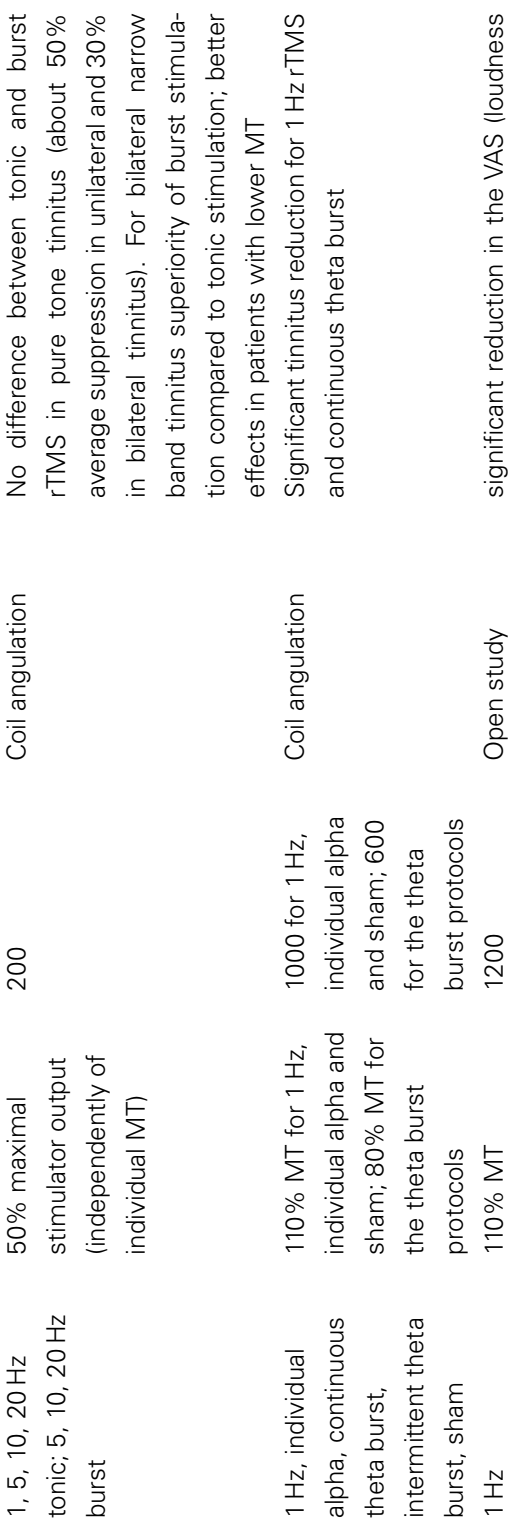

perception. The best transient tinnitus suppression was achieved by using higher stimulation frequencies for tinnitus of recent onset and lower frequencies for tinnitus of longer duration. Patients who had their tinnitus for a shorter duration experienced the best results (De Ridder et al., 2005). One study (Londero et al., 2006) demonstrated reliable tinnitus suppression in only 1 out of 13 subjects after a single session of $10 \mathrm{~Hz}$ rTMS, whereas 5 out of 8 reported tinnitus suppression after $1 \mathrm{~Hz}$ rTMS. Dose-dependent effects were observed in one study, where single sessions of lowfrequency $(1 \mathrm{~Hz})$ rTMS were applied to areas of altered blood flow during lidocaine injection (Plewnia et al., 2007a). With longer lasting stimulation sessions a longer lasting tinnitus reduction was observed.

Repetitive TMS can be applied in tonic and burst mode. Bursts of three stimuli at a frequency of $50 \mathrm{~Hz}$ (interval of $20 \mathrm{~ms}$ between each stimulus), applied every $200 \mathrm{~ms}$ ( $5 \mathrm{~Hz}$, Theta burst) have been shown to induce more pronounced and longer lasting effects on the human motor cortex than tonic stimulation (Huang et al., 2005). Single sessions of continuous theta burst stimulation (three pulses at $50 \mathrm{~Hz}$, repeated at $200 \mathrm{~ms}$ intervals for up to 600 pulses for $40 \mathrm{~s}$ ) over the temporal cortex in tinnitus patients did only result in short lasting reduction of tinnitus loudness, comparable to effects achieved with single sessions of tonic stimulation, whereas other theta burst protocols had no effect at all (Poreisz et al., 2009; Lorenz et al., 2010). In two other studies single sessions of burst stimulation were compared with tonic stimulation (De Ridder et al., 2007c,d). Burst stimulation had similar effects as tonic stimulation in patients with pure tone tinnitus but was superior in patients with noise-like tinnitus. It was hypothesized that pure tone tinnitus may be due to increased neuronal activity in the classical (lemniscal) tonotopically organized auditory pathways, which mainly fire tonically, whereas noise-like tinnitus may be the result of increased activity in the non-classical (extralemniscal) non- (or less) tonotopically organized auditory pathways, characterized by burst firing (Hu et al., 1994; De Ridder et al., 2010).

Even if single studies indicate some relationship between specific tinnitus characteristics, stimulation parameters, and behavioral effects, available data are by far not sufficient to draw firm conclusions about such relationships. An unspecific effect by the acoustic artifact can be largely excluded since practically all studies controlled for this confounding factor, e.g., by using a sham coil that produces the same sound like the real coil. In contrast the involvement of peripheral stimulation of somatosensory afferents cannot be entirely excluded. Comparison of rTMS effects and effects of transcutaneous electrical stimulation (TENS) at the neck in the same patient group demonstrate a relationship between response to these two interventions which might serve as a hint for either the involvement of unspecific effects or for the involvement of peripheral somatosensory nerve structures in the mediation of the rTMS effect. However, these peripheral or unspecific effects do not explain the entire rTMS effect (Vanneste et al., 2011b).

In summary the available data provide evidence that interference with temporal or temporoparietal cortex by single sessions of rTMS have a transient effect on the tinnitus percept in about half of the stimulated patients. 
Repeated sessions of rTMS. In 21 studies with a total of over 600 participants the effects of repeated sessions of rTMS over temporal or temporoparietal areas have been investigated (Table 2). Among these studies 10 randomized placebo-controlled trials with 234 participants are counted. Most rTMS treatment studies applied low-frequency rTMS in long trains of 1200-2000 pulses repeatedly over 5-10 days. Repeated sessions of rTMS were first investigated in a placebo-controlled cross-over study with 14 participants. The site of maximum activation in the auditory cortex was determined by $\left[{ }^{18} \mathrm{~F}\right]$ deoxyglucose (FDG) PET and a neuronavigational system was used for exact placement of the TMS coil over this area (Kleinjung et al., 2005). After active treatment the participants experienced a significant decrease in their tinnitus reflected by the score of the tinnitus questionnaire, whereas sham treatment showed no effect. Treatment effects were still detectable 6 months after treatment. Another study concerned the effects of 2 weeks of rTMS applied over the cortical area where lidocaine-induced activity change was largest as determined by $\left[{ }^{15} \mathrm{O}\right] \mathrm{H}_{2} \mathrm{O}$ PET (Plewnia et al., 2007b). This approach also resulted in moderate but significant effects after active stimulation. Placing the coil over the left temporal area according to the 10-20 EEG coordinate systems (Langguth et al., 2006b) also resulted in a significant reduction of tinnitus severity after 10 sessions of $1 \mathrm{~Hz}$ rTMS. Beneficial effects of low-frequency rTMS have been confirmed by many (Rossi et al., 2007; Smith et al., 2007; Khedr et al., 2008, 2010; Anders et al., 2010; Marcondes et al., 2010; Chung et al., 2011; Mennemeier et al., 2011) but not by all controlled studies (Piccirillo et al., 2011). Unspecific effects can largely be excluded since all mentioned studies controlled for the acoustic artifact by using a sham condition and in two recent studies the control condition even involved additional electrical superficial nerve stimulation (Rossi et al., 2007; Mennemeier et al., 2011). The degree of improvement and the duration of treatment effects varied across the studies. This may be due to differences in study design, outcome variables, stimulation parameters, selection criteria of the participants, and the stimulation target. In this context it should be mentioned that the exact cortical region in which temporal rTMS exerts clinical effects in tinnitus patients is still a matter of debate (Langguth et al., 2010). It has been argued that the primary auditory cortex is difficult to reach by TMS since it is located far from the brain surface in the Sylvian fissure in lateromedial direction. Furthermore, following the tonotopic organization of the primary auditory cortex the representation of low frequencies is located more lateral whereas the representation of high frequencies resides more medial. Thus one would expect better outcomes in patients with low-frequent tinnitus since the related abnormalities in the auditory cortex are expected to be more lateral and should therefore be better reached by rTMS. However such a relationship could not be demonstrated (Frank et al., 2010). It has been proposed that rTMS might exert direct effects on the superficial secondary auditory cortex which then further propagate to the primary auditory cortex, analogously to what has been described for electrical stimulation of the secondary auditory cortex in tinnitus (De Ridder et al., 2004).

In summary most studies investigating rTMS over temporal or temporoparietal cortical areas found a statistically significant reduction of tinnitus complaints. However, the available data do not provide a hint for superiority of specific stimulation paradigms or stimulation targets. Since most studies assessed treatment effects only by questionnaires which do not differentiate between changes in tinnitus loudness and tinnitus annoyance it is also not clear whether rTMS reduces primarily tinnitus loudness, tinnitus annoyance, or both.

Neuronal correlates of rTMS effects on tinnitus. The above mentioned studies which assessed the behavioral effect of rTMS over auditory brain areas on tinnitus perception could demonstrate that interfering with neuronal activity in these areas results in a reduction in tinnitus perception. However, they do not provide information about which neuronal changes are related to these perceptual changes. Such information can be obtained by performing neuroimaging studies before and after brain stimulation. The observed changes of neuronal activity can be related to the behavioral changes and differentiate (1) between state and trait related changes and (2) between neuronal alterations in tinnitus patients that are really causally relevant for tinnitus, unrelated to tinnitus, or even represent a beneficial compensatory mechanism.

Moreover the identification of neuronal mechanisms of rTMS induced tinnitus reduction is essential for developing optimization strategies for rTMS treatment (Kleinjung and Langguth, 2009). Unfortunately the number of studies investigating neuronal correlates of rTMS treatment is still relatively limited.

In one study voxel based morphometry and auditory evoked potentials were used to investigate the effects of TMS over the auditory cortex in healthy controls. An increase in the N2-P2 amplitude was found after active rTMS and transient structural alterations in the temporal cortex and in the thalamus (May et al., 2007). No comparison with behavioral effects can be made, since the study was performed in healthy controls, in which no behavioral changes were observed. Nevertheless the results indicate that structural changes are not automatically a trait marker, but could also serve as a state marker, since they can be induced by 1 week of rTMS. The observed changes in the thalamus and the stimulated cortical area were interpreted as a hint for an influence of rTMS on thalamocortical processing. This interpretation has been further supported by electrophysiologic studies both in healthy controls (Eichhammer et al., 2007) and in tinnitus patients (Langguth et al., 2008) before and after stimulation. By investigating motor cortex excitability it has been shown that the cortical silent period, a marker for inhibitory thalamic function, increased after stimulation (Eichhammer et al., 2007) and that this increase was related to improvement of tinnitus (Langguth et al., 2008).

One study used single photon emission computed tomography (SPECT) for exploring neuronal changes induced by five sessions of left temporoparietal low-frequency $(1 \mathrm{~Hz})$ rTMS (Marcondes et al., 2010). Comparison of SPECT data before and 2 weeks after active rTMS revealed no change in the directly stimulated area, but reduction of neuronal activity in both the left and the right temporal lobe and increased activity in the right uncus and the right cingulate gyrus. In contrast, sham rTMS resulted in increased activity in the left temporal lobe, the cingulated gyrus bilaterally, and in the right insula.

In a recent study FDG-PET scans were performed before and after five sessions of active and sham rTMS (Mennemeier 


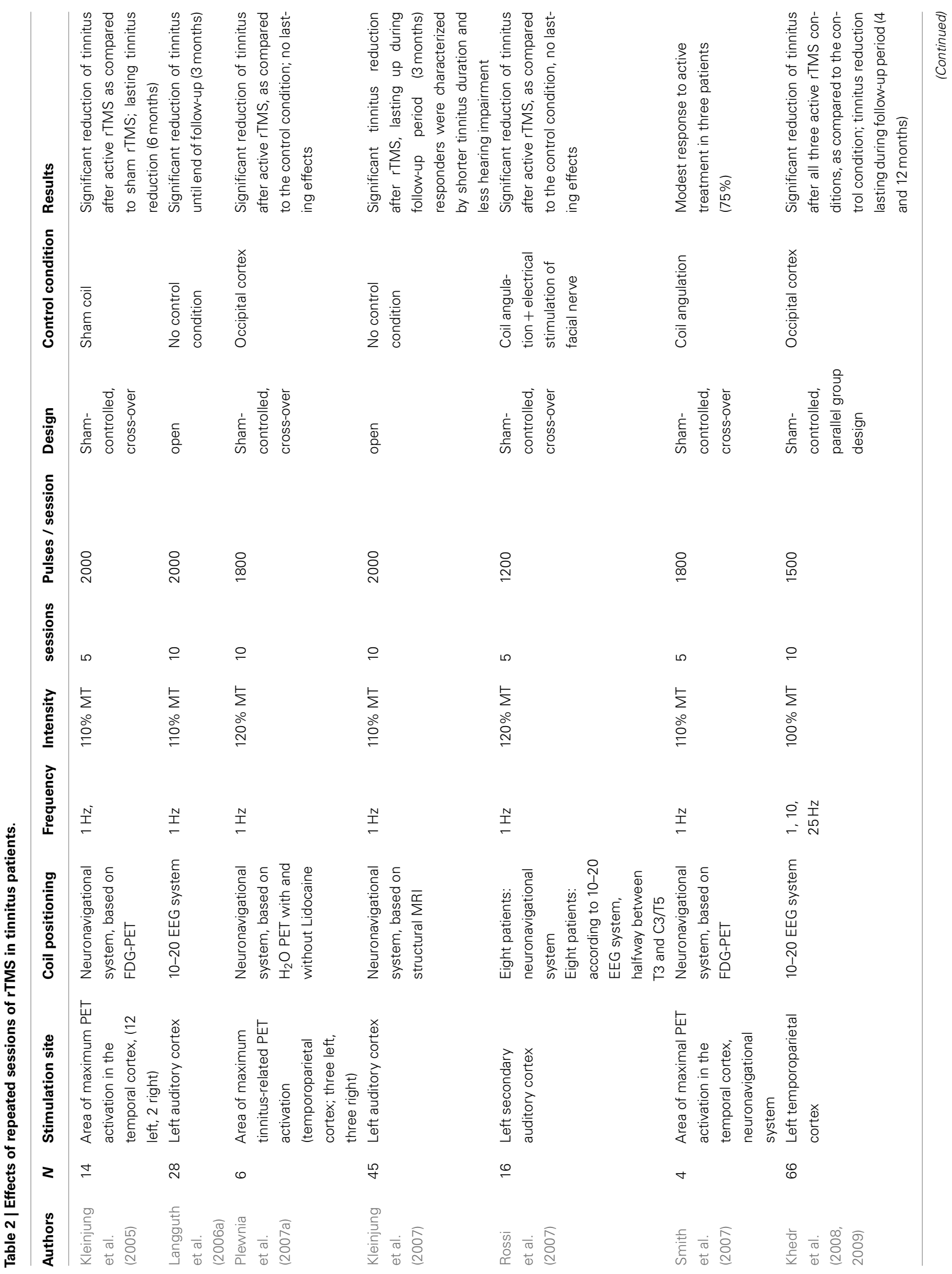




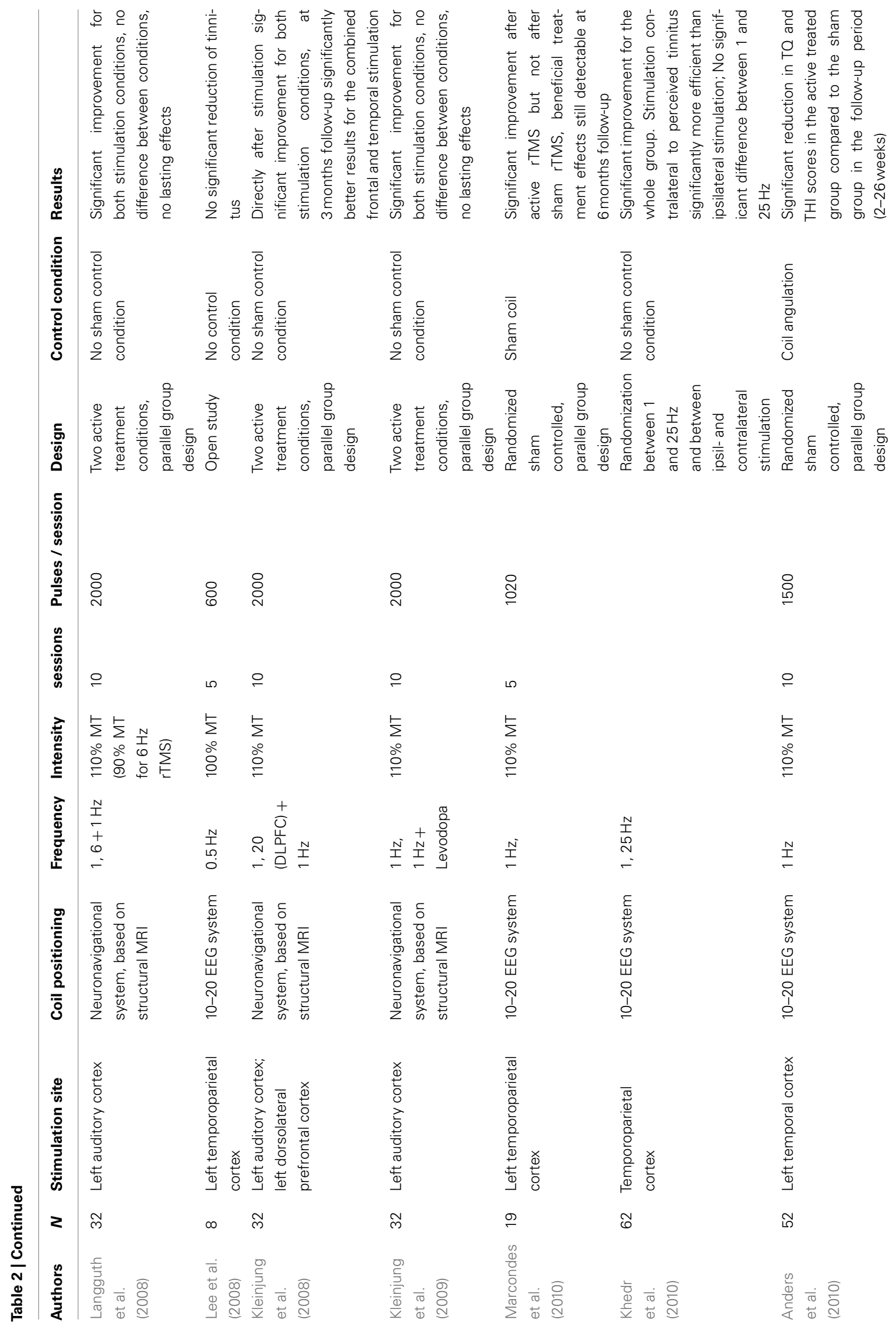



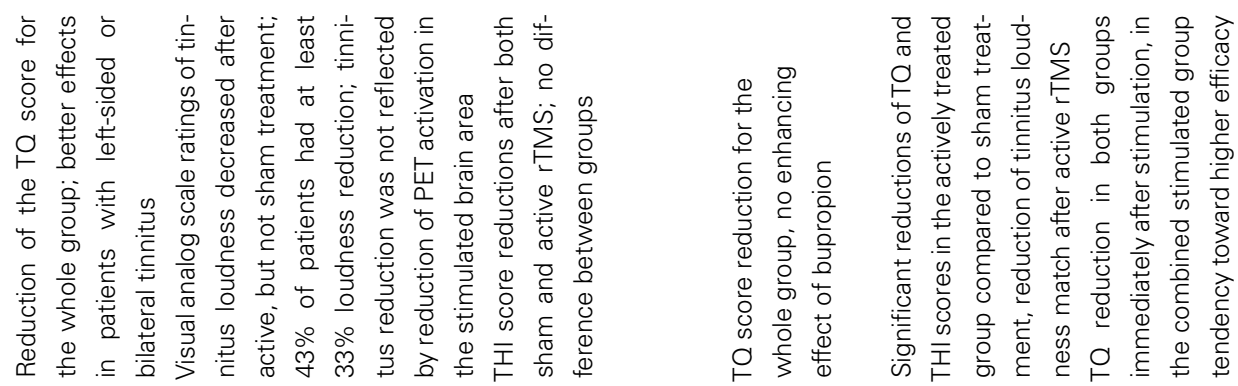

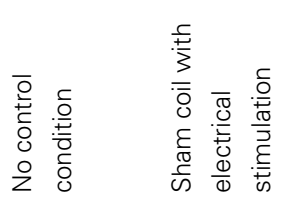

$\frac{\varepsilon}{0}$
$\frac{d}{\omega}$
$\frac{0}{\omega}$
$\overline{0}$
0
$\frac{\varepsilon}{0}$
$\frac{c}{\omega}$
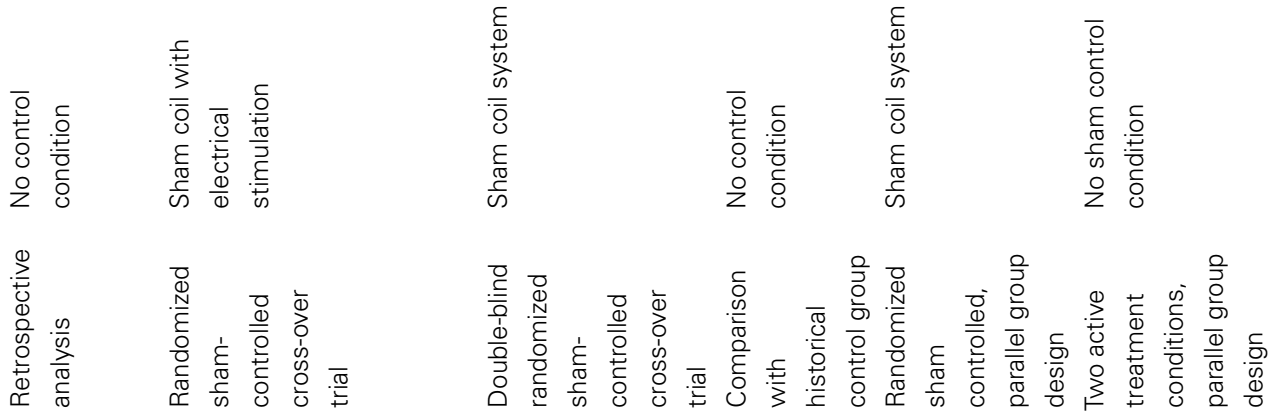

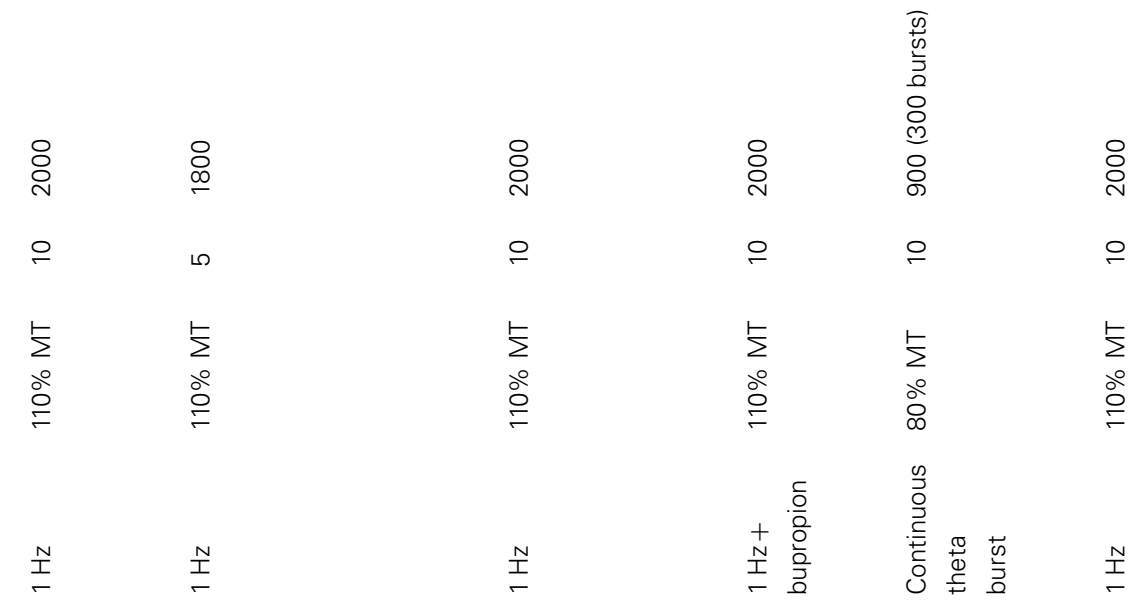
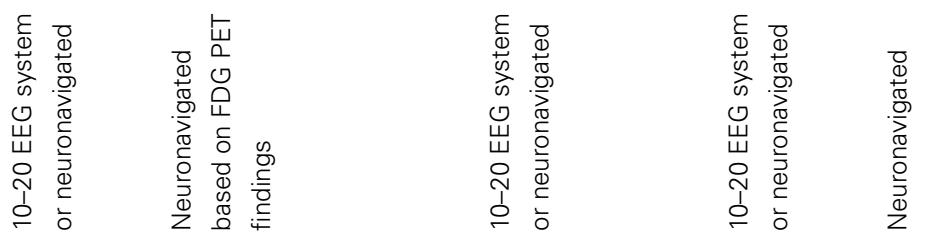

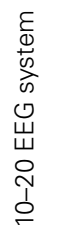
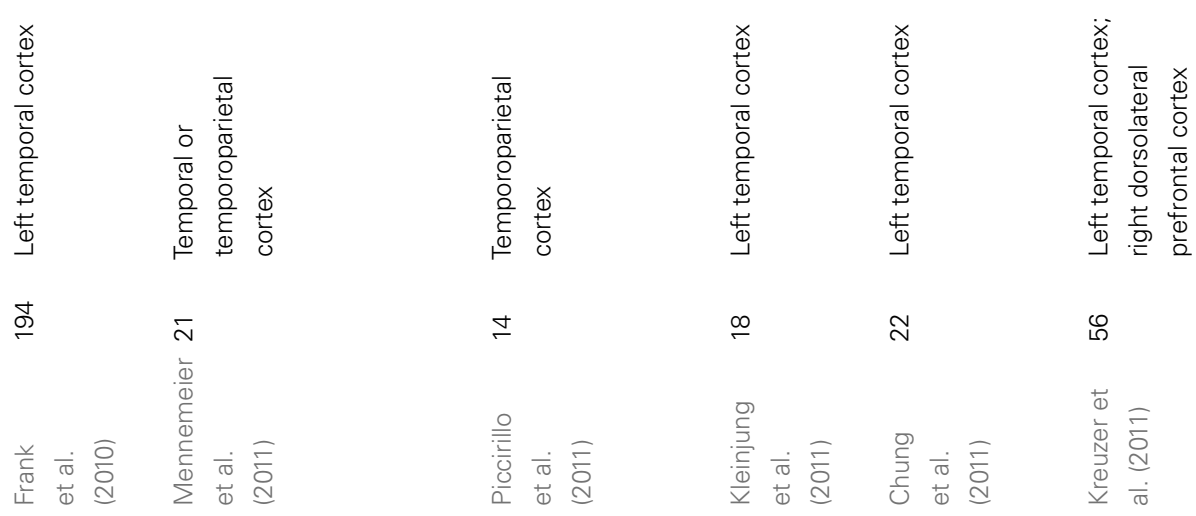
et al., 2011). The site most consistently associated with a positive response was the secondary auditory cortex either hemisphere. Whereas PET activity decreased significantly beneath the stimulating coil following active treatment, similar changes occurred at control sites and after sham stimulation. Moreover no relationship between the treatment related change of metabolic activation of the auditory cortex and clinical effects could be detected, indicating that FDG-PET does not represent a sensitive method for identifying the neuronal correlates of rTMS induced tinnitus reduction and probably also not useful for defining the stimulation target (Mennemeier et al., 2011).

Another recent study used MEG to record auditory evoked potentials of three different tones before and after five different stimulation protocols in patients with tinnitus $(1 \mathrm{~Hz}$, stimulation at the individual alpha frequency, continuous theta burst, intermittent theta burst, sham; Lorenz et al., 2010). An important finding was that the effect of the different protocols varied from patient to patient. On average, the $1-\mathrm{Hz}$ protocol revealed the most pronounced tinnitus reduction, but in individual patients other protocols turned out to be more efficient. The most consistent electrophysiological finding was a significant reduction of the auditory steady-state response (aSSR) after rTMS, indicating significant changes of neuronal activity in the directly stimulated auditory cortex ipsilateral to the coil placement. The reduction of the aSSR also correlated significantly with the perceived reduction of tinnitus loudness. Interestingly a similar relationship has been observed in a study where tinnitus reduction was achieved by a specific auditory stimulation (Okamoto et al., 2010). Importantly, the reduction of the aSSR was not related to the TMS intervention, but to the reduction of tinnitus induced by TMS.

The rTMS effect on the N1 depended on the frequency of the tone for which the auditory evoked response was assessed with inverse effects for low and high-frequency tones. Different explanations may account for this frequency-specific effect. First the cortical representations of the tested high-, middle-, and lowfrequency tones have different localizations in the auditory cortex and may as such be reached by TMS differently. A second explanation may relate to the dependency of rTMS effects on the activity of the stimulated cortical area. Animal models of tinnitus have demonstrated differential effects of noise trauma induced tinnitus on auditory cortex excitability. Excitability in the deafferentiated area is characterized by reduced inhibitory function, whereas the normal hearing region exhibits increased inhibitory and excitatory transmission (Yang et al., 2011). Thus the differential effects of rTMS on the N1 of the different tones may reflect differential TMS effects due to differences in the excitability state of different areas in the auditory cortex of tinnitus patients.

Resting state measurements before and after the different rTMS protocols revealed a correlation between tinnitus reduction and reduction of gamma activity and increase in alpha activity (Müller et al., submitted). Similar like for the aSSR measurements these effects were only observed when rTMS resulted in a reduction of tinnitus loudness.

Thus in summary there are only few studies that assessed TMS effects over the auditory cortex on both behavioral and neuronal level. Available data suggest, that rTMS modulates thalamocortical activity and that the neuronal effects are rather related to the behavioral effects of rTMS than to the rTMS protocol itself. Thus the same rTMS protocol can have different effects on the neuronal activity of a stimulated patient. However if rTMS is able to induce specific effects on neuronal activity, this is accompanied by a reduction in tinnitus loudness.

\section{Transcranial direct current stimulation}

Based on findings of increased neuronal activity in the auditory cortex of tinnitus patients tDCS over the left temporoparietal cortex has been investigated by two studies involving relatively small sample sizes ( $N=7$, Fregni et al., 2004 and $N=20$, Garin et al., 2011). In both studies single sessions of anodal tDCS applied over the left temporoparietal area and with the cathode placed contralateral over the supraorbital area resulted in a transient reduction of tinnitus, whereas no effect was found from a single session of cathodal tDCS applied over the left temporoparietal area. These findings are remarkable since anodal tDCS is assumed to increase cortical excitability. In some patients these effects lasted for several days (Garin et al., 2011). No studies with repeated applications of tDCS over auditory brain areas have been performed and there are also no data available that provide information about neuronal effects of temporal tDCS in tinnitus patients.

\section{Stimulation of the auditory cortex with implanted electrodes}

Neuronal activity in the auditory cortex can be also modified by direct electrical stimulation via implanted electrodes. In contrast to rTMS which can only be applied for a limited amount of time electrical stimulation via implanted electrodes can be performed permanently.

Clinical effects of stimulation of the auditory cortex with implanted electrodes. The largest sample derives from the TRI Tinnitus Clinic in Antwerp, Belgium, where 43 patients with severe treatment resistant tinnitus were implanted with a cortical electrode overlying the secondary auditory cortex (De Ridder et al., 2011c). Patients were only eligible to implantation when TMS over the auditory cortex resulted in a placebo-controlled suppression of the tinnitus on two separate occasions. Although all patients reacted to TMS, one out of three patients did not respond to the cortical stimulation after implantation. Among the responders to cortical stimulation there was an average decrease in the perceived tinnitus loudness of $51.3 \%$. There was a significant but weak positive correlation $(r=0.34, p<0.05)$ between the amount of the suppression effect from the preceding test TMS and cortical stimulation after implantation (De Ridder et al., 2011c).

With respect to epidural stimulation protocols, it has been observed that burst stimulation (five stimuli of $1 \mathrm{~ms}$ pulse width, $1 \mathrm{~ms}$ interpulse interval delivered at $500 \mathrm{~Hz}, 40$ times a second) is more efficient than tonic stimulation. With tonic stimulation only one in three patients responded to stimulation. With burst stimulation half of the non-responding patients did benefit, resulting in a total response rate of two out of three patients. Burst stimulation was specifically superior to tonic stimulation for suppressing noise-like tinnitus (De Ridder et al., 2011c). Furthermore, treatment effects depended on tinnitus type. Pure tone tinnitus can be suppressed better than narrow band noise or the combination of pure tone and narrow band noise, and unilateral tinnitus better than bilateral tinnitus. This approach has been replicated by 
other centers. A French study obtained long-lasting 65\% tinnitus reduction in a woman using a fMRI based extradural auditory cortex implant (Litre et al., 2009, 2010). Another study of eight patients using a similar technique but different hardware found no permanent tinnitus suppression (Friedland et al., 2007). In six out of the eight patients, temporary effects on tinnitus perception were observed. However, tinnitus distress decreased slowly over time, even without suppression of tinnitus intensity. This may be related to the fact that an electrode with only two contacts was used which limits the way the electrodes can be programmed. The finding of decreased tinnitus distress with unchanged tinnitus intensity could possibly be explained by disruption of phase synchronization between the "general distress network" and the tinnitus-related activity in the auditory cortex (De Ridder et al., 2008). Since the reduction of tinnitus distress in that study (Friedland et al., 2007) occurred slowly during the follow-up period, which was not anymore sham controlled non-specific effects cannot be ruled as well. Intracortical microstimulation in the auditory cortex of animals not only disrupts local ongoing activity but also affects long-range connections in a larger network (Deliano et al., 2009), which is similar to findings in humans using TMS of the auditory cortex (Langguth et al., 2008; May et al., 2007).

In four patients an intradural electrode on the primary auditory cortex was inserted in the Sylvian fissure, stimulating gray matter of the primary auditory cortex (De Ridder et al., 2004, 2006a). In two patients the purpose was to obtain stabilization of tinnitus suppression, because the stimulus parameters had to be reprogrammed every 2-3 days. In both patients the intradural positioning resulted in a stabilized suppression of their tinnitus. However, in the two patients who did not respond at all to epidural stimulation the intradural extracerebral stimulation was not beneficial either.

Also wire electrodes have been inserted in the primary auditory cortex, with comparable results (De Ridder et al., 2007a; Seidman et al., 2008).

Neuronal effects of stimulation of the auditory cortex with implanted electrodes. MEG during electrical stimulation of the auditory cortex revealed that the electrical stimulation increased spectral correlation across low and high gamma band activity and between alpha and beta activity, whereas delta/theta activity decreased, suggesting that auditory cortex stimulation affects thalamocortical dysrhythmia (Ramirez et al., 2009). This has been confirmed by recordings from electrodes overlying the secondary auditory cortex. Maximal tinnitus suppression was obtained by current delivery exactly at the BOLD spot, identified by tinnitusmatched sound presentation during fMRI. Recordings from this electrode revealed increased gamma and theta activity in contrast to the other electrode poles. These spectral changes normalize when stimulation induces tinnitus suppression, both on electrode and source localized EEG recordings (De Ridder et al., 2011b). Furthermore, only at the BOLD area autocorrelations showed theta-gamma coupling. These findings are in line with the hypothesis that changes in the theta- and gamma-frequency band may be causally related to a conscious auditory phantom percept as proposed by the model of thalamocortical dysrhythmia (Llinas et al., 1999; De Ridder et al., 2011b). Thus in summary EEG, MEG, and recordings from the implanted electrode support the notion that electrical stimulation reduces tinnitus perception by interfering with the abnormal thalamocortical dysrhythmia embedded in a larger tinnitus network.

Electrical stimulation of the auditory cortex has also been investigated in animal experiments where it significantly suppressed behavioral evidence of noise induced tinnitus and enhanced hearing detection (Zhang et al., 2011). In contrast auditory cortex stimulation did not induce behavioral changes in animals that did not manifest any behavioral evidence of tinnitus following the same noise exposure. These results have been interpreted in the sense that electrical stimulation of the auditory cortex may involve restoration of abnormal central auditory processing (Zhang et al., 2011).

\section{THE RELEVANCE OF NON-AUDITORY BRAIN AREAS FOR TINNITUS}

It is assumed that the activation in the auditory system is necessary but not sufficient for causing an auditory percept. Activation in the auditory system only becomes conscious if it is synchronously connected to larger co-activated "awareness" and "salience" brain networks consisting of the inferior parietal cortex, the dorsolateral prefrontal cortex, the anterior cingulated cortex (ACC), anterior insula, and the posterior cingulate cortex (PCC). Thus analogous to the global workspace model elaborated for the visual system (Dehaene et al., 2006; Baars and Franklin, 2007) and recently extended to the auditory system (Bekinschtein et al., 2009; Pegado et al., 2010), the function of the primary sensory cortices may be mainly to generate an appropriate neural discriminatory representation of the sensory input, but tinnitus only becomes conscious when activity in the auditory cortex becomes functionally connected to a network of higher order brain areas (De Ridder et al., 2011a; see Figure 1). This is in line with the neuroimaging literature that demonstrates involvement of both auditory and non-auditory areas in tinnitus (for an overview see Table 3; Adjamian et al., 2009; Lanting et al., 2009). Tinnitus-related neural networks overlap with brain regions involved in attention (Doesburg et al., 2012) to and processing of normal sounds, and in auditory memory (Schulze et al., 2011), including the primary and secondary auditory cortex, the anterior cingulate, the dorsolateral prefrontal, and the parietal cortex. Tinnitus-related brain activity also overlaps with brain areas activated by aversive sound stimulation (Mirz et al., 2000), those related to the reward and emotional system, such as nucleus accumbens, insula, and amygdala and to the hippocampal area related to memory (Langguth et al., 2011).

Tinnitus distress may then be reflected by a simultaneously co-activated distress network consisting of the anterior cingulate cortex, anterior insula, and amygdale (De Ridder et al., 2006b, 2011; Schlee et al., 2008; Vanneste et al., 2010b). This distress network is non-specific and is similarly activated in chronic pain or somatoform disorders (Landgrebe et al., 2008). An oversensitivity of this network as consequence of sensory discrimination impairment has been proposed as an additional factor in the pathogenesis of tinnitus (Landgrebe et al., 2009a).

Memory mechanisms may play a role in the persistence of the awareness of the phantom percept as well as in the reinforcement 
of the associated distress. Hippocampal involvement has been documented in animal models of tinnitus (Goble et al., 2009; Kraus et al., 2010) as well as by neuroimaging (Landgrebe et al., 2009b; Vanneste et al., 2011d). Accordingly, invasive supraselective amytal injection in the anterior choroidal artery, supplying the amygdalohippocampal area, resulted in transient reduction of the pure tone

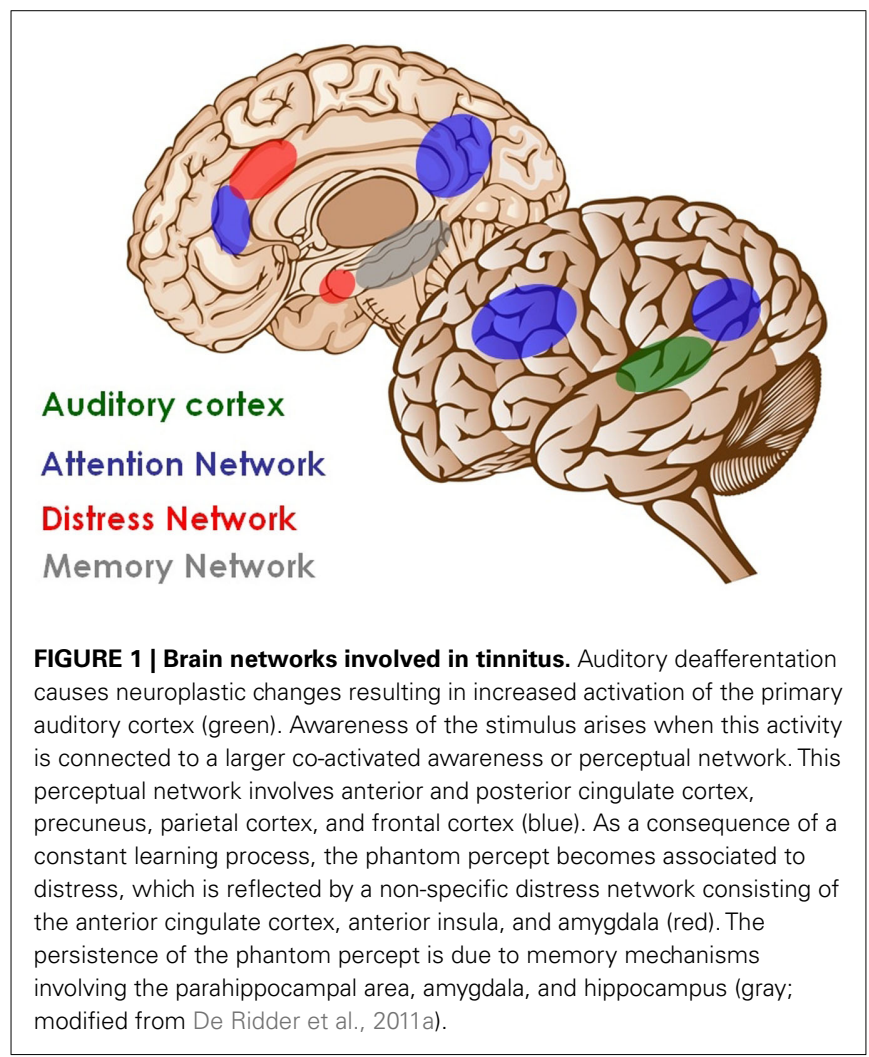

component of the contralateral tinnitus loudness (De Ridder et al., 2006b). There is an important mutual interaction between the different involved networks which may be relevant for the maintenance of tinnitus even after disappearance of the initial trigger. As an example, it has been proposed that tinnitus may be the result of a deficient sensory attentional gating mechanism originating in the subgenual cingulate cortex/nucleus accumbens area and acting on the reticular thalamic nucleus thereby modulating thalamocortical processing in the auditory system (Rauschecker et al., 2010).

Pilot data suggest that the generators involved in tinnitus of recent onset seem to change over time with increased activity in several brain areas [auditory cortex, supplementary motor area, and dorsal anterior cingulate cortex (dACC) plus insula], associated with changes in connectivity between the different auditory and non-auditory brain structures. This is so both for EEG recordings (Vanneste et al., 2011e) and MEG recordings (Schlee et al., 2009). The MEG study looking at phase-locked connectivity in the tinnitus network found that in patients with a tinnitus history of less than 4 years, the left temporal cortex is predominant in the gamma band network, whereas this network is more widely distributed, including more frontal and parietal regions, in patients with tinnitus duration of more than 4 years (Schlee et al., 2009). The EEG study demonstrates a decrease of overall connectivity with increasing tinnitus duration (Vanneste et al., 2011e). An exception to this general connectivity decrease is an increase in gamma-band connectivity between the left primary and secondary auditory cortex and the left insula, and also between the auditory cortices and the right dorsolateral prefrontal cortex. These networks are both connected to the left parahippocampal area (Vanneste et al., 2011e). Thus both studies find that acute and chronic tinnitus are related to differential activity and connectivity in a network comprising the auditory cortices, insula, dACC, and premotor cortex. A recent FDG-PET study confirmed that the

Table 3 | Functional imaging studies in individuals with tinnitus: synopsis of results.

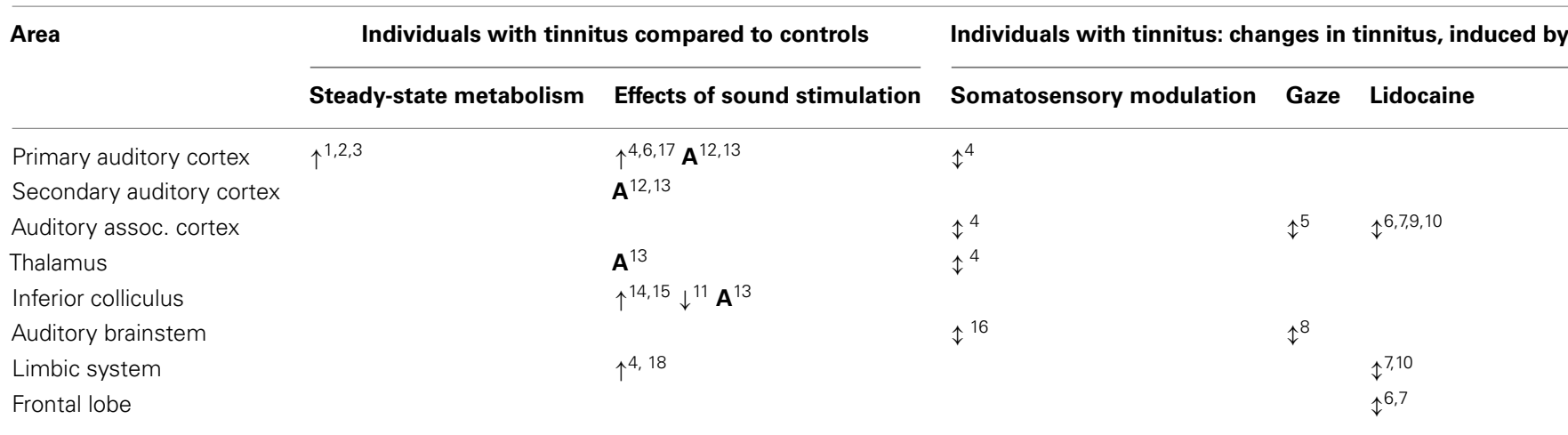

$\uparrow$, increased asymmetry of FDG uptake.

$\downarrow$, increased response to sound: reduced response to sound.

$\downarrow$, increased and reduced rCBF corresponding to increased and reduced tinnitus.

A, abnormal assymetry.

Studies: FDG-PET: ${ }^{1}$ Arnold et al. (1996), ${ }^{2}$ Wang et al. (2001), ${ }^{3}$ Langguth et al. (2006a); H2 O-PET: ${ }^{4}$ Lockwood et al. (1998), ${ }^{5}$ Giraud et al. (1999), ${ }^{6}$ Mirz et al. (1999), ${ }^{7}$ Mirz et al. (2000), ${ }^{8}$ Lockwood et al. (2001), ${ }^{9}$ Reyes et al. (2002), ${ }^{10}$ Plewnia et al. (2007a); fMRI: " ${ }^{1}$ Melcher et al. (2000), ${ }^{12}$ Kovacs et al. (2006), ${ }^{13}$ Smits et al. (2007), ${ }^{14}$ Lanting et al. (2008), ${ }^{15}$ Melcher et al. (2009), ${ }^{16}$ Lanting et al. (2010), ${ }^{17} \mathrm{Gu}$ et al. (2010), ${ }^{18}$ Leaver et al. (2011). 
relative contribution of the different brain networks depends on tinnitus characteristics such as tinnitus distress or tinnitus duration (Schecklmann et al., 2011a). Thus, in summary the functional neuroimaging literature converges in the finding that tinnitus is related to functional and structural alterations in auditory and non-auditory brain areas involving an architecture of interacting and separable tinnitus-related subnetworks (De Ridder et al., 2011a; see Figure 1).

\section{MODULATION OF NON-AUDITORY BRAIN AREAS}

Findings of the involvement of non-auditory areas in tinnitus are limited by the correlational approach of neuroimaging which cannot inform about the real relevance of the observed alterations. Hypotheses about a causal relation can be tested by interfering with the activity in the various identified brain areas and investigating changes in tinnitus perception or annoyance. Compared to the stimulation of auditory areas much less data are available.

\section{Effects of rTMS of non-auditory brain areas}

The effects of rTMS over the dorsolateral prefrontal cortex (DLPFC), the dorsal anterior cingulated cortex (dACC), the ventrolateral prefrontal cortex (VLPFC), and the intraparietal sulcus (IPS) have been investigated. Results are summarized in Table 4 (single sessions) and Table 5 (repeated sessions). All mentioned studies only investigated behavioral effects of stimulation. Thus there is no information available about the neuronal changes underlying the reported behavioral changes in tinnitus patients.

Single sessions of rTMS over the dorsolateral prefrontal cortex (DLPFC). As mentioned, activity in the auditory cortex has to be linked to other "global workspace" areas such as the dorsolateral prefrontal cortex (DLPFC) in order to gain access to consciousness. The DLPFC seems to play a specific role in auditory processing.
The DLPFC has a bilateral facilitatory effect on auditory memory storage and contains auditory memory cells (Bodner et al., 1996). The DLPFC also exerts early inhibitory modulation of input to primary auditory cortex in humans (Knight et al., 1989) and has been found to be associated with auditory attention (Alain et al., 1998; Voisin et al., 2006) resulting in top-down modulation of auditory processing (Mitchell et al., 2005). In order to test the involvement of the DLPFC in tinnitus it has been investigated whether rTMS of the DLPFC results in tinnitus suppression. In this study in 62 patients $1 \mathrm{~Hz}$ rTMS (200 pulses) over the right DLPFC resulted in a significant reduction of tinnitus loudness as compared to sham stimulation (Vanneste et al., unpublished data; Table 4). These data enlarge the knowledge from imaging studies by indicating the critical involvement of the right DLPFC in the pathophysiology of a subgroup of tinnitus patients.

Repeated sessions of rTMS with combined stimulation over the DLPFC and the temporal cortex. There is no published study reporting results from repeated sessions of rTMS of the DLPFC alone. However both high-frequency left rTMS and low-frequency right rTMS over the DLPFC have been combined with lowfrequency rTMS over the left temporal cortex (Table 5). It has also been shown that left DLPFC stimulation followed by auditory cortex TMS results in better tinnitus suppression after 3 months than isolated auditory cortex stimulation (Kleinjung et al., 2008). These results could be confirmed in a large controlled trial (Langguth et al., submitted) and in a retrospective analysis of 47 patients who were treated with the combined protocol as compared to 188 patients who were stimulated only over the left temporal cortex only (Burger et al., 2011). In a recent study left temporal stimulation combined with low-frequency rTMS over the right DLPFC showed also a trend toward more pronounced effects as compared to temporal stimulation alone (Kreuzer et al., 2011).

Table 4 | Effects of single sessions of rTMS over non-auditory brain areas.

\begin{tabular}{|c|c|c|c|c|c|c|c|c|}
\hline Authors & $N$ & $\begin{array}{l}\text { Stimulation } \\
\text { site }\end{array}$ & $\begin{array}{l}\text { Coil } \\
\text { positioning }\end{array}$ & Frequency & Intensity & $\begin{array}{l}\text { Pulses/ } \\
\text { session }\end{array}$ & $\begin{array}{l}\text { Control } \\
\text { condition }\end{array}$ & Results \\
\hline $\begin{array}{l}\text { Vanneste } \\
\text { (submitted) }\end{array}$ & 62 & Right DLPFC & $\begin{array}{l}\text { 10-20 EEG } \\
\text { system }\end{array}$ & $11 \mathrm{~Hz}$ & $90 \% \mathrm{MT}$ & 200 & Coil angulation & $\begin{array}{l}\text { Significant VAS reduction/10\% } \\
\text { reduction for group, } 35 \% \text { for } \\
\text { responders. } 56 \% \text { of patients } \\
\text { respond }\end{array}$ \\
\hline $\begin{array}{l}\text { Vanneste } \\
\text { et al. } \\
\text { (2011c) }\end{array}$ & 78 & $\begin{array}{l}\text { Bifrontal } \\
\text { targeting } \\
\text { dACC }\end{array}$ & $\begin{array}{l}\text { Anatomical } \\
\text { landmarks: } \\
1.5 \mathrm{~cm} \text { anterior } \\
\text { to } 1 / 3 \text { of the } \\
\text { distance from } \\
\text { the nasion- inion }\end{array}$ & $\begin{array}{l}1,3,5,10 \\
20 \mathrm{~Hz}\end{array}$ & $\begin{array}{l}50 \% \text { machine } \\
\text { output }\end{array}$ & 200 & Coil angulation & $\begin{array}{l}52 \text { placebo-negative ( } 21 \text { non- } \\
\text { responders, } 31 \text { responders): } \\
1 \& 3 \mathrm{~Hz} \text { significantly better } \\
\text { than sham, } 5 \mathrm{~Hz} \text { equal to sham, } \\
10 \& 20 \mathrm{~Hz} \text { worse than sham. }\end{array}$ \\
\hline $\begin{array}{l}\text { Vanneste } \\
\text { (submitted) }\end{array}$ & 60 & Left VLPFC & $\begin{array}{l}\text { Anatomical } \\
\text { landmarks }\end{array}$ & $\begin{array}{l}1 \text { and } \\
10 \mathrm{~Hz}\end{array}$ & $90 \% \mathrm{MT}$ & 200 & Coil angulation & $\begin{array}{l}\text { Only } 10 \mathrm{~Hz} \text { better than sham. } \\
35 \text { placebo negative. } 22 \% \\
\text { improvement for group, } 37 \% \\
\text { for responders. }\end{array}$ \\
\hline \multirow[t]{2}{*}{$\begin{array}{l}\text { Vanneste } \\
\text { (submitted) }\end{array}$} & $\begin{array}{l}64 \\
(40+24)\end{array}$ & $\begin{array}{l}\text { Bilateral IPS } \\
\text { (40 patients) }\end{array}$ & $\begin{array}{l}\text { Anatomical } \\
\text { landmarks }\end{array}$ & $1,5,10 \mathrm{~Hz}$ & $\begin{array}{l}50 \% \text { of } \\
\text { maximal } \\
\text { stimulator }\end{array}$ & 200 & Coil angulation & $\begin{array}{l}\text { Only } 5 \& 10 \mathrm{~Hz} \text { significant for } \\
\text { bilateral IPS: } 9 \% \text { improvement. }\end{array}$ \\
\hline & & $\begin{array}{l}\text { Left IPS (24 } \\
\text { patients) }\end{array}$ & & & output & & & $\begin{array}{l}\text { Only } 10 \mathrm{~Hz} \text { significant for left IPS: } \\
10 \% \text { improvement }\end{array}$ \\
\hline
\end{tabular}


Table 5 | Effects of repeated sessions of rTMS over non-auditory brain areas.

\begin{tabular}{|c|c|c|c|c|c|c|c|c|c|c|}
\hline Authors & $N$ & $\begin{array}{l}\text { Stimulation } \\
\text { site }\end{array}$ & $\begin{array}{l}\text { Coil } \\
\text { positioning }\end{array}$ & Frequency & Intensity & sessions & $\begin{array}{l}\text { Pulses/ } \\
\text { session }\end{array}$ & Design & $\begin{array}{l}\text { Control } \\
\text { condition }\end{array}$ & Results \\
\hline $\begin{array}{l}\text { Kleinjung } \\
\text { et al. } \\
\text { (2008) }\end{array}$ & 32 & $\begin{array}{l}\text { Left auditory } \\
\text { cortex; left } \\
\text { dorsolateral } \\
\text { prefrontal } \\
\text { cortex }\end{array}$ & $\begin{array}{l}\text { neuron } \\
\text { avigational } \\
\text { system, } \\
\text { based on } \\
\text { structural } \\
\text { MRI }\end{array}$ & $\begin{array}{l}1 \mathrm{~Hz} \\
20 \mathrm{~Hz} \\
(\mathrm{DLPFC})+ \\
1 \mathrm{~Hz}\end{array}$ & $110 \% \mathrm{MT}$ & 10 & 2000 & $\begin{array}{l}\text { Two active } \\
\text { treatment } \\
\text { conditions, } \\
\text { parallel group } \\
\text { design }\end{array}$ & $\begin{array}{l}\text { No sham } \\
\text { control } \\
\text { condition }\end{array}$ & $\begin{array}{l}\text { Directly after stimulation } \\
\text { significant improvement } \\
\text { for both stimulation } \\
\text { conditions, at } 3 \text { months } \\
\text { follow-up significantly } \\
\text { better results for the } \\
\text { combined frontal and } \\
\text { temporal stimulation }\end{array}$ \\
\hline $\begin{array}{l}\text { Kreuzer } \\
\text { et al., } \\
2011\end{array}$ & 56 & $\begin{array}{l}\text { Left temporal } \\
\text { cortex; right } \\
\text { dorsolateral } \\
\text { prefrontal } \\
\text { cortex }\end{array}$ & $\begin{array}{l}\text { 10-20 EEg } \\
\text { system }\end{array}$ & $1 \mathrm{~Hz}$ & $110 \% \mathrm{MT}$ & 10 & 2000 & $\begin{array}{l}\text { Two active } \\
\text { treatment } \\
\text { conditions, } \\
\text { parallel group } \\
\text { design }\end{array}$ & $\begin{array}{l}\text { No sham } \\
\text { control } \\
\text { condition }\end{array}$ & $\begin{array}{l}\text { TQ reduction in both } \\
\text { groups immediately } \\
\text { after stimulation, in the } \\
\text { combined stimulated } \\
\text { group tendency toward } \\
\text { higher efficacy }\end{array}$ \\
\hline
\end{tabular}

rTMS of the dorsal anterior cingulated cortex with the double cone coil. Resting state EEG and MEG recordings in tinnitus patients have shown that the dorsal part of the anterior cingulate cortex is involved both in chronification of tinnitus (Schlee et al., 2009; Vanneste et al., 2011d) as well as in tinnitus-related distress (Weisz et al., 2005a; Vanneste et al., 2010b; De Ridder et al., 2011). The use of a double cone coil permits to modulate the activity in the dorsal part of the anterior cingulate cortex as demonstrated by a PET study (Hayward et al., 2007). In a study on 78 tinnitus patients it was shown that 1 and $3 \mathrm{~Hz}$ of DCC frontal TMS can improve both tinnitus intensity and tinnitus distress, $5 \mathrm{~Hz}$ is equal to sham and $20 \mathrm{~Hz}$ is significantly worse than sham (Vanneste et al., 2011c; Table 4). Of 78 tinnitus patients, 52 had no response to sham stimulation. Of these 52 sham negative participants, 31 patients were TMS responders. For this latter group the mean reduction of the VAS score for tinnitus intensity was $34.38 \%$, for tinnitusrelated distress $26 \%$. These findings confirm the relevance of the dACC for tinnitus intensity and distress in a substantial part of the investigated population.

TMS of the ventrolateral prefrontal cortex. It has recently been proposed that tinnitus may be a compensatory mechanism related to incongruity emerging from a discrepancy between an expected sound and the perceived sound that is distorted due to cochlear impairment (De Ridder et al., 2011a). Visual incongruity is known to involve the ventrolateral prefrontal cortex, the anterior cingulate cortex, the supplementary motor area, and the inferior parietal area (Michelon et al., 2003). Thus a study was initiated in 60 patients targeting the left VLPFC for tinnitus suppression at 1 and $10 \mathrm{~Hz}$ (Vanneste et al., submitted; Table 4). $1 \mathrm{~Hz}$ TMS was no better than sham, but $10 \mathrm{~Hz}$ had a significant effect on tinnitus. Among those participants who did not respond to sham $10 \mathrm{~Hz}$ over the VLPFC suppressed tinnitus perception in average by 21.9 and by $36.7 \%$ when only responders were analyzed. Of interest is the frequency dependent effect for stimulation of the left VLPFC, which is contrary to the DLPFC where only $1 \mathrm{~Hz}$ revealed a reduction of tinnitus perception.
rTMS of the parietal cortex. The extremely emotional context of disabling tinnitus often leads to a higher level of selective attention directed toward the tinnitus. As such, tinnitus is a continuously distracting auditory event. Auditory attention activates the intrapartietal sulcus (IPS), and modulating the IPS with $10 \mathrm{~Hz}$ TMS creates the ability to ignore salient distractors (Mevorach et al., 2010). Thus it is expected that modulating the IPS may interfere with the perception of tinnitus. The effect of TMS on tinnitus has recently been evaluated using a double cone coil overlying the left IPS in 24 individuals (study 1) and in 40 individuals with the double cone coil symmetrically overlying both IPS areas (study 2; Vanneste et al., submitted; Table 4). In study 1 patients reported a significant transient reduction of the tinnitus percept after $10 \mathrm{~Hz}$ stimulation in comparison to pre-treatment, sham, and $1 \mathrm{~Hz}$ stimulation, respectively, with a suppression effect of $11.36 \%$. No significant effect was obtained for $1 \mathrm{~Hz}$ stimulation with the coil tilted toward the left IPS. In study 2 patients revealed a significant suppression effect on 1,5 , and $10 \mathrm{~Hz}$ in comparison to pre-treatment. However, only stimulation at 5 and $10 \mathrm{~Hz}$ had a significant difference in comparison to sham with a suppression effect of respectively 8.78 and $9.50 \%$. These data suggest that the IPS is involved in tinnitus perception and that $10 \mathrm{~Hz}$ TMS using the double cone coil overlying the IPS can modulate tinnitus, predominantly via the left IPS.

\section{Effects of tDCS of non-auditory brain areas}

Several studies have used tDCS to interfere with tinnitus by modulation of activity in the dorsolateral prefrontal cortex (DLPFC; Vanneste et al., 2010a; Faber et al., 2011; Vanneste and De Ridder, 2011; Frank et al., 2012). In a large study involving 448 individuals with non-pulsatile tinnitus, it was shown that a single session of tDCS with the anode over the right DLPFC and the cathode over the left DLPFC could cause tinnitus suppression in $29.9 \%$ of the participants (Vanneste et al., 2010a). In contrast the opposite stimulation procedure with the cathode over the right DLPFC and the anode over the left DLPFC had no effects in a sample of 30 patients (Vanneste et al., 2010a). Six repeated bifrontal tDCS sessions 
within 3 weeks with the cathode over the left DLPFC and the anode over the right DLPFC reduced tinnitus loudness, unpleasantness, and discomfort especially in female tinnitus patients (Frank et al., 2012). In a double-blind, placebo-controlled cross-over study 15 subjects with tinnitus were randomly assigned to active and sham anodal tDCS over left $(N=8)$ or right $\operatorname{DLPFC}(N=7)$ for six sessions in a counterbalanced order, with the cathode electrode placed on the contralateral DLPFC. Both active conditions, irrespective of the anodal position decreased tinnitus annoyance but not tinnitus intensity. Furthermore, anodal stimulation of the left DLPFC had a significant effect on depression, whereas anodal stimulation of the right DLPFC reduced symptoms of anxiety (Faber et al., 2011).

In conclusion, these preliminary studies indicate that both anodal stimulation of the left auditory cortex and bifrontal tDCS with the cathode left and the anode right can have beneficial effects on tinnitus in some individuals. The interindividual variability of treatment effects is high in all studies, suggesting that there may be pathophysiologically distinct forms of tinnitus that respond particularly well to different tDCS protocols (Vanneste et al., 2011c).

In order to unravel the mechanism by which tDCS suppresses tinnitus EEG measurements were performed before and after single sessions of bifrontal tDCS in 12 patients who responded to tDCS. Reduction of tinnitus intensity and tinnitus-related distress was related to modulation of neuronal activity in pregenual anterior cingulate cortex, parahippocampal area, and right primary auditory cortex regions (Vanneste et al., 2011a). These findings are comparable to those obtained in healthy controls after a similar tDCS intervention (anode positioned over the left DLPFC and the cathode over the right supraorbital region), that revealed a tDCS induced modulation of regional electrical activity in the left subgenual prefrontal cortex, the anterior cingulate cortex and the left parahippocampus (Keeser et al., 2011b) and significant changes of regional brain connectivity both for the default mode network and the fronto-parietal network (Keeser et al., 2011a).

\section{Effects of direct electric stimulation of non-auditory brain areas}

In a few tinnitus patients also implanted electrodes have been used to stimulate non-auditory brain areas. In one patient with intractable tinnitus electrodes have been implanted over the DLPFC (De Ridder et al., 2011d). Knowledge about the effects of DBS derives from patients who received DBS for movement disorders and suffered from comorbid tinnitus.

Effects of epidural electrodes over the DLPFC. In a patient intractable to conservative medical management and TMS of the auditory cortex, a neuronavigation-based auditory fMRI-guided frontal cortex TMS session was performed in a placebo-controlled way, yielding 50\% tinnitus suppression. Based on the same concept of epidural auditory cortex stimulation and motor cortex stimulation, this TMS result was used as a predictive indication to implant two extradural electrodes (De Ridder et al., 2011d). The exact localization was determined by the $\mathrm{AMRI-BOLD}$ response in the DLPFC during presentation of a sound that was matched to the individual tinnitus sound. Postoperatively the tinnitus immediately improved by $66.67 \%$ and has progressively continued to improve for more than 1 year. The initial VAS of $8 / 10$ has decreased after 1 year to $2 / 10$. This suggests that in selected patients focal extradural electrical stimulation of the dorsolateral prefrontal cortex at the area of tinnitus-matched sound elected BOLD activation is capable of suppressing contralateral tinnitus partially.

Effects of deep brain stimulation. Whereas rTMS, tDCS, and epidural stimulation exert their effects primarily in superficial brain regions, DBS can modulate brain activity very focally in deeper brain regions. To our knowledge there are no published reports of patients who received DBS for the treatment of tinnitus. However, results are available from patients who received DBS in the ventralis intermedius nucleus of the thalamus (Shi et al., 2009) or in the caudate nucleus (Cheung and Larson, 2010) for movement disorders who also reported having tinnitus.

In one study, seven patients implanted with DBS systems for movement disorders who also reported having tinnitus were interviewed and asked about their tinnitus conditions. Three of the seven patients reported reduced tinnitus loudness when DBS was turned on. Of the four patients tested in a specialized tinnitus clinic, results indicated that DBS of the ventralis intermedius nucleus of the thalamus caused decreases in tinnitus loudness in two patients with relatively prolonged residual inhibition (Shi et al., 2009).

The caudate is routinely traversed during DBS implantation of the subthalamic nucleus and ventral intermediate nucleus in awake patients for treatment of Parkinson's disease and essential tremor, respectively. In six tinnitus patients who underwent DBS for movement disorders. the effect of DBS in the locus of caudate neurons (area LC) was evaluated with respect to the patients' tinnitus (Cheung and Larson, 2010). In five subjects tinnitus loudness in both ears was suppressed to an intensity level $2 / 10$ or less. In one subject, where the DBS lead was outside the area LC, tinnitus was not modulated. Hearing thresholds were unchanged by area LC stimulation.

These results suggest that DBS of non-auditory thalamus and caudate structures may provide tinnitus relief for some patients. The mechanisms involved in tinnitus suppression by DBS are yet unknown, but it has been suggested that stimulation of area LC of the caudate nucleus may modulate auditory gating function (Larson and Cheung, 2012).

\section{CONCLUSION}

Imaging studies demonstrate that tinnitus is related to structural and functional alterations in multiple brain structures including auditory cortex, thalamus, dorsal, and subgenual anterior and posterior cingulated cortex, ventromedial prefrontal, and dorsolateral prefrontal cortex, parietal cortex, precuneus, insula, amygdale, hippocampus, and parahippocampus. The findings from imaging studies are complemented by brain stimulation studies that demonstrate reduction of tinnitus loudness and/or distress after stimulation of temporal, temporoparietal, parietal, dorsolateral and ventromedial prefrontal cortex, and ACC.

It has been suggested that different aspects of tinnitus such as perceptual aspects (loudness, tone-, or noise-like, laterality), attention, salience, distress, mood, memory, and duration are reflected by the involvement of specific networks (De Ridder et al., 2011a), a claim which is currently only partly supported by empirical evidence from imaging or brain stimulation studies. This may also 
be due to methodological difficulties related to (1) reliable assessment of the different aspects of tinnitus, (2) the need for large samples in order to differentiate the neuronal correlate of specific aspects, (3) the limited resolution and sensitivity of the currently used imaging techniques, and (4) incomplete understanding of the mechanisms of action of the various brain stimulation techniques.

In spite of these constraints the available data demonstrate that focal modulation of neuronal activity by brain stimulation techniques provides a useful complementary approach to neuroimaging, which is limited by its strictly correlational character. Important added value can be obtained by investigating the effects of focal modulation of the different areas showing up in functional imaging studies of tinnitus. Assessment of neuronal

\section{REFERENCES}

Adjamian, P., Sereda, M., and Hall, D. A. (2009). The mechanisms of tinnitus: perspectives from human functional neuroimaging. Hear. Res. 253, 15-31.

Alain, C., Woods, D. L., and Knight, R. T. (1998). A distributed cortical network for auditory sensory memory in humans. Brain Res. 812, 23-37.

Anders, M., Dvorakova, J., Rathova, L., Havrankova, P., Pelcova, P., Vaneckova, M., Jech, R., Holcat, M., Seidl, Z., and Raboch, J. (2010). Efficacy of repetitive transcranial magnetic stimulation for the treatment of refractory chronic tinnitus: a randomized, placebo controlled study. Neuro Endocrinol. Lett. 31, 238-249.

Arnold, W., Bartenstein, P., Oestreicher, E., Romer, W., and Schwaiger, M. (1996). Focal metabolic activation in the predominant left auditory cortex in patients suffering from tinnitus: a PET study with [18F]deoxyglucose. ORL J. Otorhinolaryngol. Relat. Spec. 58, 195-199.

Attias, J., Furman, V., Shemesh, Z., and Bresloff, I. (1996). Impaired brain processing in noise-induced tinnitus patients as measured by auditory and visual event-related potentials. Ear Hear. 17, 327-333.

Attias, J., Urbach, D., Gold, S., and Shemesh, Z. (1993). Auditory event related potentials in chronic tinnitus patients with noise induced hearing loss. Hear. Res. 71, 106-113.

Baars, B. J., and Franklin, S. (2007). An architectural model of conscious and unconscious brain functions: global workspace theory and IDA. Neural Netw. 20, 955-961.

Barker, A. T., Jalinous, R., and Freeston, I. L. (1985). Non-invasive magnetic stimulation of human motor cortex. Lancet 1, 1106-1107.

Bekinschtein, T. A., Dehaene, S., Rohaut, B., Tadel, F., Cohen, L., and Naccache, L. (2009). Neural signature of the conscious processing of auditory regularities. Proc. Natl. Acad. Sci. U.S.A. 106, 1672-1677.

Bodner, M., Kroger, J., and Fuster, J. M. (1996). Auditory memory cells in dorsolateral prefrontal cortex. $\mathrm{Neu}$ roreport 7, 1905-1908.

Borchers, S., Himmelbach, M., Logothetis, N., and Karnath, H. O. (2011). Direct electrical stimulation of human cortex - the gold standard for mapping brain functions? Nat. Rev. Neurosci. 3, 63-70.

Burger, J., Frank, E., Kreuzer, P., Kleinjung, T., Vielsmeier, V., Landgrebe, M., Hajak, G., and Langguth, B. (2011). Transcranial magnetic stimulation for the treatment of tinnitus: 4-year follow-up in treatment responders-a retrospective analysis. Brain Stimul. 4, 222-227.

Cheung, S. W., and Larson, P. S. (2010). Tinnitus modulation by deep brain stimulation in locus of caudate neurons (area LC). Neuroscience 169, 1768-1778.

Chung, H. K., Tsai, C. H., Lin, Y. C., Chen, J. M., Tsou, Y. A., Wang, C. Y., Lin, C. D., Jeng, F. C., Chung, J. G., and Tsai, M. H. (2011). Effectiveness of theta-burst repetitive transcranial magnetic stimulation for treating chronic tinnitus. Audiol. Neurootol. 17, 112-120.

De Ridder, D., De Mulder, G., Verstraeten, E., Seidman, M., Elisevich, K., Sunaert, S., Kovacs, S., Van der Kelen, K., Van de Heyning, P., and Moller, A. (2007a). Auditory cortex stimulation for tinnitus. Acta Neurochir. Suppl. 97, 451-462.

De Ridder, D., De Mulder, G., Verstraeten, E., Sunaert, S., and Moller, A. (2007b). Somatosensory cortex stimulation for deafferentation pain. Acta Neurochir. Suppl. 97, 67-74.

De Ridder, D., van der Loo, E., Van der Kelen, K., Menovsky, T., Van de Heyning, P., and Moller, A. (2007c).

changes related to tinnitus reduction or worsening can reveal important information about which neuronal changes are directly related to tinnitus and which reflect predisposition, compensatory changes, or epiphenomena. Thus, correlations between brain activity and/or connectivity and tinnitus can tentatively be turned into causal relationships. This can be performed for tinnitus as a unified percept, or for specific aspects of tinnitus (e.g., distress, depression, loudness,... .) if well controlled for other aspects. This will ultimately lead to a better understanding of which areas or subnetworks are critically involved in the generation of each aspect of tinnitus and ultimately in tinnitus as a unified percept. The pathophysiological neural correlates could subsequently provide important information for improving brain stimulation techniques as a treatment strategy.

Do tonic and burst TMS modulate the lemniscal and extralemniscal system differentially? Int. J. Med. Sci. 4, 242-246.

De Ridder, D., van der Loo, E., Van der Kelen, K., Menovsky, T., Van de Heyning, P., and Moller, A. (2007d). Theta, alpha and beta burst transcranial magnetic stimulation: brain modulation in tinnitus. Int. J. Med. Sci. 4, 237-241.

De Ridder, D., De Mulder, G., Verstraeten, E., Van der Kelen, K., Sunaert, S., Smits, M., Kovacs, S., Verlooy, J., Van de Heyning, P., and Moller, A. R. (2006a). Primary and secondary auditory cortex stimulation for intractable tinnitus. ORL J. Otorhinolaryngol. Relat. Spec. 68, 48-54.

De Ridder, D., Fransen, H., Francois, O., Sunaert, S., Kovacs, S., and Van de Heyning, P. (2006b). Amygdalohippocampal involvement in tinnitus and auditory memory. Acta Otolaryngol. Suppl. 556, 50-53.

De Ridder, D., De Mulder, G., Walsh, V., Muggleton, N., Sunaert, S., and Moller, A. (2004). Magnetic and electrical stimulation of the auditory cortex for intractable tinnitus. Case report. J. Neurosurg. 100, 560-564.

De Ridder, D., Elgoyhen, A. B., Romo, R., and Langguth, B. (2011a). Phantom percepts: tinnitus and pain as persisting aversive memory networks. Proc. Natl. Acad. Sci. U.S.A. 108, 8075-8080.

De Ridder, D., van der Loo, E., Vanneste, S., Gais, S., Plazier, M., Kovacs, S., Sunaert, S., Menovsky, T., and Van de Heyning, P. (2011b). Theta-gamma dysrhythmia and auditory phantom perception. J. Neurosurg. 114, 912 921.

De Ridder, D., Vanneste, S., Kovacs, S., Sunaert, S., Menovsky, T., Van de Heyning, P., and Moller, A. (2011c). Transcranial magnetic stimulation and extradural electrodes implanted on secondary auditory cortex for tinnitus suppression. J. Neurosurg. 114, 903-911.

De Ridder, D., Vanneste, S., Plazier, M., Menovsky, T., Van de, H. P., Kovacs, S., and Sunaert, S. (2011d). Dorsolateral prefrontal cortex transcranial magnetic stimulation and electrode implant for intractable tinnitus. World Neurosurg. PMID: 22120273. [Epub ahead of print].

De Ridder, D., Menovsky, T., and Van de Heyning, P. (2008). Auditory cortex stimulation for tinnitus suppression. Otol. Neurotol. 29, 574-575.

De Ridder, D., Vanneste, S., and Congedo, M. (2011). The distressed brain: a group blind source separation analysis on tinnitus. PLoS ONE 6, e24273. doi:10.1371/journal.pone. 0024273

De Ridder, D., Vanneste, S., van der Loo, E., Plazier, M., Menovsky, T., and Van de Heyning, P. (2010). Burst stimulation of the auditory cortex: a new form of neurostimulation for noiselike tinnitus suppression. J. Neurosurg. 112, 1289-1294.

De Ridder, D., Verstraeten, E., Van der, K. K., De Mulder, G., Sunaert, S., Verlooy, J., Van de, H. P., and Moller, A. (2005). Transcranial magnetic stimulation for tinnitus: influence of tinnitus duration on stimulation parameter choice and maximal tinnitus suppression. Otol. Neurotol. 26, 616-619.

Dehaene, S., Changeux, J. P., Naccache, L., Sackur, J., and Sergent, C. (2006). Conscious, preconscious, and subliminal processing: a testable taxonomy. Trends Cogn. Sci. (Regul. Ed.) 10, 204-211.

Deliano, M., Scheich, H., and Ohl, F. W. (2009). Auditory cortical activity after intracortical microstimulation and its role for sensory processing and learning. J. Neurosci. 29, 15898-15909. 
Doesburg, S. M., Green, J. J., McDonald, J. J., and Ward, L. M. (2012). Theta modulation of interregional gamma synchronization during auditory attention control. Brain Res. 1431, 77-85.

Dymond, A. M., Coger, R. W., and Serafetinides, E. A. (1975). Intracerebral current levels in man during electrosleep therapy. Biol. Psychiatry 10, 101-104.

Eggermont, J. J., and Roberts, L. E. (2004). The neuroscience of tinnitus. Trends Neurosci. 27, 676-682.

Eichhammer, P., Kleinjung, T., Landgrebe, M., Hajak, G., and Langguth, B. (2007). TMS for treatment of chronic tinnitus: neurobiological effects. Prog. Brain Res. 166, 369-375.

Faber, M., Vanneste, S., Fregni, F., and De, R. D. (2011). Top down prefrontal affective modulation of tinnitus with multiple sessions of tDCS of dorsolateral prefrontal cortex. Brain Stimul. doi: 10.1016/j.brs.2011.09.003. [Epub ahead of print].

Folmer, R. L., Carroll, J. R., Rahim, A., Shi, Y., and Hal Martin, W. (2006). Effects of repetitive transcranial magnetic stimulation (rTMS) on chronic tinnitus. Acta Otolaryngol. Suppl. 556, 96-101.

Frank, E., Schecklmann, M., Landgrebe, M., Burger, J., Kreuzer, P., Poeppl, T. B., Kleinjung, T., Hajak, G., and Langguth, B. (2012). Treatment of chronic tinnitus with repeated sessions of prefrontal transcranial direct current stimulation: outcomes from an openlabel pilot study. J. Neurol. 259, 327-333.

Frank, G., Kleinjung, T., Landgrebe, M., Vielsmeier, V., Steffenhagen, C., Burger, J., Frank, E., Vollberg, G., Hajak, G., and Langguth, B. (2010). Left temporal low-frequency rTMS for the treatment of tinnitus: clinical predictors of treatment outcome a retrospective study. Eur. J. Neurol. 17, 951-956.

Fregni, F., Santos, C. M., Myczkowski, M. L., Rigolino, R., Gallucci-Neto, J., Barbosa, E. R., Valente, K. D., Pascual-Leone, A., and Marcolin, M. A. (2004). Repetitive transcranial magnetic stimulation is as effective as fluoxetine in the treatment of depression in patients with Parkinson's disease. J. Neurol. Neurosurg. Psychiatr. 75, 1171-1174.

Fregni, F., Marcondes, R., Boggio, P. S., Marcolin, M. A., Rigonatti, S. P., Sanchez, T. G., Nitsche, M. A., and Pascual-Leone, A. (2006). Transient tinnitus suppression induced by repetitive transcranial magnetic stimulation and transcranial direct current stimulation. Eur. J. Neurol. 13, 996-1001.

Friedland, D. R., Gaggl, W., RungeSamuelson, C., Ulmer, J. L., and Kopell, B. H. (2007). Feasibility of auditory cortical stimulation for the treatment of tinnitus. Otol. Neurotol. 28, 1005-1012.

Garin, P., Gilain, C., Van Damme, J. P., de, F. K., Jamart, J., Ossemann, M., and Vandermeeren, Y. (2011). Short- and long-lasting tinnitus relief induced by transcranial direct current stimulation. J. Neurol. 258, 1940-1948.

George, M. S., and ston-Jones, G. (2010). Noninvasive techniques for probing neurocircuitry and treating illness: vagus nerve stimulation (VNS), transcranial magnetic stimulation (TMS) and transcranial direct current stimulation (tDCS). Neuropsychopharmacology 35, 301-316.

Giraud, A. L., Chéry-Croze, S., Fischer, G., Fischer, C., Vighetto, A., Grégoire, M. C., Lavenne, F., and Collet, L. (1999). A selective imaging of tinnitus. Neuroreport 10, 1-5.

Goble, T. J., Moller, A. R., and Thompson, L. T. (2009). Acute high-intensity sound exposure alters responses of place cells in hippocampus. Hear. Res. 253, 52-59.

Gu, J. W., Halpin, C. F., Nam, E. C., Levine, R. A., and Melcher, J. R. (2010). Tinnitus, diminished soundlevel tolerance, and elevated auditory activity in humans with clinically normal hearing sensitivity. $J$. Neurophysiol. 104, 3361-3370.

Hallett, M. (2000). Transcranial magnetic stimulation and the human brain. Nature 406, 147-150.

Hayward, G., Mehta, M. A., Harmer, C., Spinks, T. J., Grasby, P. M., and Goodwin, G. M. (2007). Exploring the physiological effects of doublecone coil TMS over the medial frontal cortex on the anterior cingulate cortex: an H2(15)O PET study. Eur. J. Neurosci. 25, 2224-2233.

Hoffman, R. E., and Cavus, I. (2002). Slow transcranial magnetic stimulation, long-term depotentiation, and brain hyperexcitability disorders. Am. J. Psychiatry 159, 1093 1102.

House, J. W., and Brackmann, D. E. (1981). Tinnitus: surgical treatment. Ciba Found. Symp. 85, 204-216.

$\mathrm{Hu}$, B., Senatorov, V., and Mooney, D. (1994). Lemniscal and nonlemniscal synaptic transmission in rat auditory thalamus. J. Physiol. (Lond.) 479(Pt 2), 217-231.
Huang, Y. Z., Edwards, M. J., Rounis, E., Bhatia, K. P., and Rothwell, J. C. (2005). Theta burst stimulation of the human motor cortex. Neuron 45, 201-206.

Keeser, D., Meindl, T., Bor, J., Palm, U., Pogarell, O., Mulert, C., Brunelin, J., Moller, H. J., Reiser, M., and Padberg, F. (2011a). Prefrontal transcranial direct current stimulation changes connectivity of resting-state networks during fMRI. J. Neurosci. 31, 15284-15293.

Keeser, D., Padberg, F., Reisinger, E., Pogarell, O., Kirsch, V., Palm, U., Karch, S., Moller, H. J., Nitsche, M. A., and Mulert, C. (2011b). Prefrontal direct current stimulation modulates resting EEG and eventrelated potentials in healthy subjects: a standardized low resolution tomography (sLORETA) study. Neuroimage 55, 644-657.

Khedr, E. M., bo-Elfetoh, N., Rothwell, J. C., El-Atar, A., Sayed, E., and Khalifa, H. (2010). Contralateral versus ipsilateral rTMS of temporoparietal cortex for the treatment of chronic unilateral tinnitus: comparative study. Eur. J. Neurol. 17, 976-983.

Khedr, E. M., Rothwell, J. C., Ahmed, M. A., and El-Atar, A. (2008). Effect of daily repetitive transcranial magnetic stimulation for treatment of tinnitus: comparison of different stimulus frequencies. J. Neurol. Neurosurg. Psychiatr. 79, 212-215.

Khedr, E. M., Rothwell, J. C., ElAtar, A. (2009). One-year follow up of patients with chronic tinnitus treated with left temporoparietal rTMS. Eur. J. Neurol. 16, 404-408.

Kleinjung, T., Eichhammer, P., Landgrebe, M., Sand, P., Hajak, G., Steffens, T., Strutz, J., and Langguth, B. (2008). Combined temporal and prefrontal transcranial magnetic stimulation for tinnitus treatment: a pilot study. Otolaryngol. Head Neck Surg. 138, 497-501.

Kleinjung, T., Eichhammer, P. Langguth, B., Jacob, P., Marienhagen, J., Hajak, G., Wolf, S. R., and Strutz, J. (2005). Long-term effects of repetitive transcranial magnetic stimulation (rTMS) in patients with chronic tinnitus. Otolaryngol. Head Neck Surg. 132, 566-569.

Kleinjung, T., Steffens, T., Landgrebe, M., Vielsmeier, V., Frank, E., Burger, J., Strutz, J., Hajak, G., and Langguth, B. (2011). Repetitive transcranial magnetic stimulation for tinnitus treatment: no enhancement by the dopamine and noradrenaline reuptake inhibitor bupropion. Brain Stimul. 4, 65-70.
Kleinjung, T., Steffens, T., Landgrebe, M., Vielsmeier, V., Frank, E., Hajak, G., Strutz, J., and Langguth, B. (2009). Levodopa does not enhance the effect of low-frequency repetitive transcranial magnetic stimulation in tinnitus treatment. Otolaryngol. Head Neck Surg. 140, 92-95.

Kleinjung, T., and Langguth, B. (2009). Strategies for enhancement of transcranial magnetic stimulation effects in tinnitus patients. Int. Tinnitus J. 15, 154-160.

Kleinjung, T., Steffens, T., Sand, P., Murthum, T., Hajak, G., Strutz, J., Langguth, B., and Eichhammer, P. (2007). Which tinnitus patients benefit from transcranial magnetic stimulation? Otolaryngol. Head Neck Surg. 137, 589-595.

Knight, R. T., Scabini, D., and Woods, D. L. (1989). Prefrontal cortex gating of auditory transmission in humans. Brain Res. 504, 338-342.

Kovacs, S., Peeters, R., Smits, M., De Ridder, D., Van Hecke, P., and Sunaert S. (2006). Activation of cortical and subcortical auditory structures at 3 $\mathrm{T}$ by means of a functional magnetic resonance imaging paradigm suitable for clinical use. Invest. Radiol. 41, 87-96.

Kraus, K. S., Mitra, S., Jimenez, Z., Hinduja, S., Ding, D., Jiang, H., Gray, L., Lobarinas, E., Sun, W., and Salvi, R. J. (2010). Noise trauma impairs neurogenesis in the rat hippocampus. Neuroscience 167, 1216-1226.

Kreuzer, P. M., Landgrebe, M., Schecklmann, M., Poeppl, T. B., Vielsmeier, V., Hajak, G., Kleinjung, T., and Langguth, B. (2011). Can temporal repetitive transcranial magnetic stimulation be enhanced by targeting affective components of tinnitus with frontal rTMS? A randomized controlled pilot trial. Front. Syst. Neurosci. 5:88. doi:10.3389/fnsys.2011.00088

Kringelbach, M. L., Jenkinson, N., Owen, S. L., and Aziz, T. Z. (2007). Translational principles of deep brain stimulation. Nat. Rev. Neurosci. 8, 623-635.

Landgrebe, M., Barta, W., Rosengarth, K., Frick, U., Hauser, S., Langguth, B., Rutschmann, R., Greenlee, M. W., Hajak, G., and Eichhammer, P. (2008). Neuronal correlates of symptom formation in functional somatic syndromes: a fMRI study. Neuroimage 41, 1336-1344.

Landgrebe, M., Frick, U., Hauser, S., Hajak, G., and Langguth, B. (2009a). Association of tinnitus and electromagnetic hypersensitivity: hints for a shared pathophysiology? PLoS 
ONE 4, e5026. doi:10.1371/journal.pone. 0005026

Landgrebe, M., Langguth, B., Rosengarth, K., Braun, S., Koch, A., Kleinjung, T., May, A., De Ridder, D., and Hajak, G. (2009b). Structural brain changes in tinnitus: grey matter decrease in auditory and nonauditory brain areas. Neuroimage 46, 213-218.

Langguth, B., Eichhammer, P., Kreutzer, A., Maenner, P., Marienhagen, J., Kleinjung, T., Sand, P., and Hajak, G. (2006a). The impact of auditory cortex activity on characterizing and treating patients with chronic tinnitus - first results from a PET study. Acta Otolaryngol. Suppl. 556, 84-88.

Langguth, B., Zowe, M., Landgrebe, M., Sand, P., Kleinjung, T., Binder, H., Hajak, G., and Eichhammer, P. (2006b). Transcranial magnetic stimulation for the treatment of tinnitus: a new coil positioning method and first results. Brain Topogr. 18, 241-247.

Langguth, B., Kleinjung, T., Landgrebe, M., De Ridder, D., and Hajak, G. (2010). rTMS for the treatment of tinnitus: the role of neuronavigation for coil positioning. Neurophysiol. Clin. 40, 45-58.

Langguth, B., Kleinjung, T., Frank, E., Landgrebe, M., Sand, P., Dvorakova, J., Frick, U., Eichhammer, P., Hajak, G. (2008). High-frequency priming stimulation does not enhance the effect of low-frequency rTMS in the treatment of tinnitus. Exp. Brain Res. 184, 587-591.

Langguth, B., Landgrebe, M., Kleinjung, T., Sand, G. P., and Hajak, G. (2011). Tinnitus and depression. World J. Biol. Psychiatry 12, 489-500.

Lanting, C. P., De, K. E., Bartels, H., and Van, D. P. (2008). Functional imaging of unilateral tinnitus using fMRI. Acta Otolaryngol. 128, 415-421.

Lanting, C. P., de Kleine, E., Eppinga, R. N., and van Dijk, P. (2010). Neural correlates of human somatosensory integration in tinnitus. Hear. Res. 267, 78-88.

Lanting, C. P., De Kleine, E., and Van Dijk, P. (2009). Neural activity underlying tinnitus generation: results from PET and fMRI. Hear. Res. 255, 1-13.

Larson, P. S., and Cheung, S. W. (2012). Deep brain stimulation in area LC controllably triggers auditory phantom percepts. Neurosurgery 70,398 405.

Leaver, A. M., Renier, L., Chevillet, M. A., Morgan, S., Kim, H. J., and Rauschecker, J. P. (2011). Dysregulation of limbic and auditory networks in tinnitus. Neuron 69, 33-43.

Lee, S. L., Abraham, M., Cacace, A. T., and Silver, S. M. (2008). Repetitive transcranial magnetic stimulation in veterans with debilitating tinnitus: a pilot study. Otolaryngol. Head Neck Surg. 138, 398-399.

Lefaucheur, J. P., Menard-Lefaucheur, I., Goujon, C., Keravel, Y., and Nguyen, J. P. (2011). Predictive value of rTMS in the identification of responders to epidural motor cortex stimulation therapy for pain. J. Pain. 12, 1102-1111.

Litre, C. F., Giersky, F., Theret, E., Leveque, M., Peruzzi, P., and Rousseaux, P. (2010). Feasibility of auditory cortical stimulation for the treatment of tinnitus. Three case reports. Neurochirurgie 56, 303-308.

Litre, C. F., Theret, E., Tran, H., Leveque, M., Portefaix, C., Gierski, F., Emeriau, S., and Peruzzi, P. (2009). Surgical treatment by electrical stimulation of the auditory cortex for intractable tinnitus. Brain Stimul. 2, 132-137.

Llinas, R. R., Ribary, U., Jeanmonod, D., Kronberg, E., and Mitra, P. P. (1999). Thalamocortical dysrhythmia: a neurological and neuropsychiatric syndrome characterized by magnetoencephalography. Proc. Natl. Acad. Sci. U.S.A. 96, 15222-15227.

Lockwood, A. H., Salvi, R. J., Coad, M. L., Towsley, M. L., Wack, D. S., and Murphy, B. W. (1998). The functional neuroanatomy of tinnitus: evidence for limbic system links and neural plasticity. Neurology 50, 114-120.

Lockwood, A. H., Wack, D. S., Burkard, R. F., Coad, M. L., Reyes, S. A., Arnold, S. A., and Salvi, R. J. (2001). The functional anatomy of gazeevoked tinnitus and sustained lateral gaze. Neurology 56, 472-480.

Londero, A., Lefaucheur, J. P., Malinvaud, D., Brugieres, P., Peignard, P., Nguyen, J. P., Avan, P., and Bonfils, P. (2006). Magnetic stimulation of the auditory cortex for disabling tinnitus: preliminary results. Presse Med. 35, 200-206.

Lorenz, I., Muller, N., Schlee, W., Langguth, B., and Weisz, N. (2010). Short-term effects of single repetitive TMS sessions on auditory evoked activity in patients with chronic tinnitus. J. Neurophysiol. 104, 1497-1505.

Marcondes, R. A., Sanchez, T. G., Kii, M. A., Ono, C. R., Buchpiguel, C. A., Langguth, B., and Marcolin, M. A. (2010). Repetitive transcranial magnetic stimulation improve tinnitus in normal hearing patients: a double-blind controlled, clinical and neuroimaging outcome study. Eur. J. Neurol. 17, 38-44.

May, A., Hajak, G., Ganssbauer, S., Steffens, T., Langguth, B., Kleinjung, T., and Eichhammer, P. (2007) Structural brain alterations following 5 days of intervention: dynamic aspects of neuroplasticity. Cereb. Cortex 17, 205-210.

Meeus, O., Blaivie, C., Ost, J., De Ridder, D., and Van de Heyning, P. (2009) Influence of tonic and burst transcranial magnetic stimulation characteristics on acute inhibition of subjective tinnitus. Otol. Neurotol. 30, 697-703.

Melcher, J. R., Levine, R. A., Bergevin, C., and Norris, B. (2009). The auditory midbrain of people with tinnitus: abnormal sound-evoked activity revisited. Hear. Res. 257, 63-74.

Melcher, J. R., Sigalovsky, I. S. Guinan, J. J. Jr., and Levine, R. A. (2000). Lateralized tinnitus studied with functional magnetic resonance imaging: abnormal inferior colliculus activation. J. Neurophysiol. 83, 1058-1072.

Mennemeier, M., Chelette, K. C., Allen, S., Bartel, T. B., Triggs, W., Kimbrell, T., Crew, J., Munn, T., Brown, G. J., and Dornhoffer, J. (2011). Variable changes in PET activity before and after rTMS treatment for tinnitus. Laryngoscope 121, 815-822.

Mevorach, C., Hodsoll, J., Allen, H., Shalev, L., and Humphreys, G. (2010). Ignoring the elephant in the room: a neural circuit to downregulate salience. J. Neurosci. 30 , 6072-6079.

Michelon, P., Snyder, A. Z., Buckner, R. L., McAvoy, M., and Zacks, J. M. (2003). Neural correlates of incongruous visual information. An event-related fMRI study. Neuroimage 19, 1612-1626.

Minami, S. B., Shinden, S., Okamoto, Y., Watada, Y., Watabe, T., Oishi, N., Kanzaki, S., Saito, H., Inoue, Y., and Ogawa, K. (2011). Repetitive transcranial magnetic stimulation (rTMS) for treatment of chronic tinnitus. Auris Nasus Larynx 38, 301306.

Mirz, F., Gjedde, A., Sodkilde-Jrgensen, H., and Pedersen, C. B. (2000). Functional brain imaging of tinnituslike perception induced by aversive auditory stimuli. Neuroreport 11, 633-637.

Mirz, F., Pedersen, B., Ishizu, K., Johannsen, P., Ovesen, T., StødkildeJørgensen, H., and Gjedde, A. (1999). Positron emission tomography of cortical centers of tinnitus. Hear. Res. 134, 133-144.

Mitchell, T. V., Morey, R. A., Inan, S., and Belger, A. (2005). Functional magnetic resonance imaging measure of automatic and controlled auditory processing. Neuroreport 16, 457-461.

Moazami-Goudarzi, M., Michels, L., Weisz, N., and Jeanmonod, D. (2010). Temporo-insular enhancement of EEG low and high frequencies in patients with chronic tinnitus. QEEG study of chronic tinnitus patients. BMC Neurosci. 11, 40. doi:10.1186/1471-2202-11-40

Nguyen, J. P., Keravel, Y., Feve, A., Uchiyama, T., Cesaro, P., Le, G. C., and Pollin, B. (1997). Treatment of deafferentation pain by chronic stimulation of the motor cortex: report of a series of 20 cases. Acta Neurochir. Suppl. 68, 54-60.

Nitsche, M. A., and Paulus, W. (2000). Excitability changes induced in the human motor cortex by weak transcranial direct current stimulation. J. Physiol. (Lond.) 527(Pt 3), 633-639.

Nitsche, M. A., and Paulus, W. (2001). Sustained excitability elevations induced by transcranial DC motor cortex stimulation in humans 3. Neurology 57, 1899-1901.

Norena, A., Cransac, H., and CheryCroze, S. (1999). Towards an objectification by classification of tinnitus. Clin. Neurophysiol. 110, 666-675.

Norena, A., Micheyl, C., Chery-Croze, S., and Collet, L. (2002). Psychoacoustic characterization of the tinnitus spectrum: implications for the underlying mechanisms of tinnitus. Audiol. Neurootol. 7, 358-369.

Norena, A. J. (2011). An integrative model of tinnitus based on a central gain controlling neural sensitivity. Neurosci. Biobehav. Rev. 35, 1089-1109.

Norena, A. J., and Eggermont, J. J. (2005). Enriched acoustic environment after noise trauma reduces hearing loss and prevents cortical map reorganization. J. Neurosci. 25, 699-705.

Okamoto, H., Stracke, H., Stoll, W., and Pantev, C. (2010). Listening to tailormade notched music reduces tinnitus loudness and tinnitus-related auditory cortex activity. Proc. Natl. Acad. Sci. U.S.A. 107, 1207-1210.

Pegado, F., Bekinschtein, T., Chausson, N., Dehaene, S., Cohen, L., and Naccache, L. (2010). Probing the lifetimes of auditory novelty detection processes. Neuropsychologia 48, 3145-3154. 
Piccirillo, J. F., Garcia, K. S., Nicklaus, J., Pierce, K., Burton, H., Vlassenko, A. G., Mintun, M., Duddy, D., Kallogjeri, D., and Spitznagel, E. L. Jr. (2011). Low-frequency repetitive transcranial magnetic stimulation to the temporoparietal junction for tinnitus. Arch. Otolaryngol. Head Neck Surg. 137, 221-228.

Pirotte, B., Neugroschl, C., Metens, T., Wikler, D., Denolin, V., Voordecker, P., Joffroy, A., Massager, N., Brotchi, J., Levivier, M., and Baleriaux, D. (2005). Comparison of functional MR imaging guidance to electrical cortical mapping for targeting selective motor cortex areas in neuropathic pain: a study based on intraoperative stereotactic navigation. AJNR Am. J. Neuroradiol. 26, 2256-2266.

Pirotte, B., Voordecker, P., Neugroschl, C., Baleriaux, D., Wikler, D., Metens, T., Denolin, V., Joffroy, A., Massager, N., Brotchi, J., and Levivier, M. (2008). Combination of functional magnetic resonance imaging-guided neuronavigation and intraoperative cortical brain mapping improves targeting of motor cortex stimulation in neuropathic pain. Neurosurgery 62(6 Suppl. 3), 941-956.

Plewnia, C., Bartels, M., and Gerloff, C. (2003). Transient suppression of tinnitus by transcranial magnetic stimulation. Ann. Neurol. 53, 263-266.

Plewnia, C., Reimold, M., Najib, A., Brehm, B., Reischl, G., Plontke, S. K., and Gerloff, C. (2007a). Dosedependent attenuation of auditory phantom perception (tinnitus) by PET-guided repetitive transcranial magnetic stimulation. Hum. Brain Mapp. 28, 238-246.

Plewnia, C., Reimold, M., Najib, A., Reischl, G., Plontke, S. K., and Gerloff, C. (2007b). Moderate therapeutic efficacy of positron emission tomography-navigated repetitive transcranial magnetic stimulation for chronic tinnitus: a randomised, controlled pilot study. J. Neurol. Neurosurg. Psychiatr. 78, 152-156.

Poreisz, C., Paulus, W., Moser, T., and Lang, N. (2009). Does a single session of theta-burst transcranial magnetic stimulation of inferior temporal cortex affect tinnitus perception? BMC Neurosci. 10, 54.doi:10.1186/1471-2202-10-54

Ramirez, R. R., Kopell, B. H., Butson, C. R., Gaggl, W., Friedland, D. R., and Baillet, S. (2009). Neuromagnetic source imaging of abnormal spontaneous activity in tinnitus patient modulated by electrical cortical stimulation. Conf. Proc.
IEEE Eng. Med. Biol. Soc. 2009, 1940-1944.

Rauschecker, J. P., Leaver, A. M., and Muhlau, M. (2010). Tuning out the noise: limbic-auditory interactions in tinnitus. Neuron 66, 819-826.

Reyes, S. A., Salvi, R. J., Burkard, R. F., Coad, M. L., Wack, D. S., Galantowicz, P. J., and Lockwood, A. H. (2002). Brain imaging of the effects of lidocaine on tinnitus. Hear. Res. 171, 43-50.

Roberts, L. E., Eggermont, J. J., Caspary, D. M., Shore, S. E., Melcher, J. R., and Kaltenbach, J. A. (2010). Ringing ears: the neuroscience of tinnitus. J. Neurosci. 30, 14972-14979.

Rosenberg, O., Roth, Y., Kotler, M., Zangen, A., and Dannon, P. (2011). Deep transcranial magnetic stimulation for the treatment of auditory hallucinations: a preliminary openlabel study. Ann. Gen. Psychiatry 10,3 .

Rossi, S., De, C. A., Ulivelli, M., Bartalini, S., Falzarano, V., Filippone, G., and Passero, S. (2007). Effects of repetitive transcranial magnetic stimulation on chronic tinnitus: a randomised, crossover, double blind, placebo controlled study. J. Neurol. Neurosurg. Psychiatr. 78, 857-863.

Santos, F. V., and Matas, C. G. (2010). Late auditory evoked potentials in individuals with tinnitus. Braz. J. Otorhinolaryngol. 76, 263-270.

Schaette, R., and Kempter, R. (2006). Development of tinnitus-related neuronal hyperactivity through homeostatic plasticity after hearing loss: a computational model. Eur. J. Neurosci. 23, 3124-3138.

Schaette, R., and McAlpine, D. (2011). Tinnitus with a normal audiogram: physiological evidence for hidden hearing loss and computational model. J. Neurosci. 31, 1345213457.

Schecklmann, M., Landgrebe, M., Poeppl, T. B., Kreuzer, P., Manner, P., Marienhagen, J., Wack, D. S., Kleinjung, T., Hajak, G., and Langguth, B. (2011a). Neural correlates of tinnitus duration and distress: a positron emission tomography study. Hum. Brain Mapp. doi: 10.1002/hbm.21426. [Epub ahead of print].

Schecklmann, M., Volberg, G., Frank, G., Hadersdorfer, J., Steffens, T., Weisz, N., Landgrebe, M., Hajak, G., Greenlee, M., Classen, J., and Langguth, B. (2011b). Paired associative stimulation of the auditory system: a proof-of-principle study. PLoS ONE 6, e27088. doi:10.1371/journal.pone.0027088
Schlee, W., Dohrmann, K., Hartmann, T., Lorenz, N., Mülller, N., Elbert, T., and Weisz, N. (2008). Assessment and modification of the tinnitusrelated cortical network. Semin. Hear. 29, 270-287.

Schlee, W., Hartmann, T., Langguth, B., and Weisz, N. (2009). Abnormal resting-state cortical coupling in chronic tinnitus. BMC Neurosci. 10 11. doi:10.1186/1471-2202-10-11

Schulze, K., Zysset, S., Mueller, K., Friederici, A. D., and Koelsch, S. (2011). Neuroarchitecture of verbal and tonal working memory in nonmusicians and musicians. Hum. Brain Mapp. 32, 771-783.

Seidman, M. D., De, R. D., Elisevich, K., Bowyer, S. M., Darrat, I., Dria, J., Stach, B., Jiang, Q., Tepley, N., Ewing, J., Seidman, M., and Zhang, J. (2008). Direct electrical stimulation of Heschl's gyrus for tinnitus treatment. Laryngoscope 118, 491-500.

Shi, Y., Burchiel, K. J., Anderson, V. C., and Martin, W. H. (2009). Deep brain stimulation effects in patients with tinnitus. Otolaryngol. Head Neck Surg. 141, 285-287.

Siebner, H. R., Filipovic, S. R., Rowe, J. B., Cordivari, C., Gerschlager, W. Rothwell, J. C., Frackowiak, R. S., and Bhatia, K. P. (2003). Patients with focal arm dystonia have increased sensitivity to slow-frequency repetitive TMS of the dorsal premotor cortex. Brain.

Smith, J. A., Mennemeier, M., Bartel, T., Chelette, K. C., Kimbrell, T., Triggs, W., and Dornhoffer, J. L. (2007). Repetitive transcranial magnetic stimulation for tinnitus: a pilot study. Laryngoscope 117, 529-534.

Smits, M., Kovacs, S., De, R. D., Peeters, R. R., Van, H. P., and Sunaert, S. (2007). Lateralization of functional magnetic resonance imaging (fMRI) activation in the auditory pathway of patients with lateralized tinnitus. Neuroradiology 49 , 669-679.

van der Loo, E., Gais, S., Congedo, M., Vanneste, S., Plazier, M., Menovsky, T., Van de Heyning, P., and De Ridder, D. (2009). Tinnitus intensity dependent gamma oscillations of the contralateral auditory cortex. PLoS ONE 4, e7396. doi:10.1371/journal.pone.0007396

Vanneste, S., and De Ridder, D. (2011). Bifrontal transcranial direct current stimulation modulates tinnitus intensity and tinnitusdistress-related brain activity. Eur. J. Neurosci. 34, 605-614.

Vanneste, S., Focquaert, F., Van de Heyning, P., and De Ridder, D. (2011a). Different resting state brain activity and functional connectivity in patients who respond and not respond to bifrontal tDCS for tinnitus suppression. Exp. Brain Res. 210 217-227.

Vanneste, S., Langguth, B., and De Ridder, D. (2011b). Do tDCS and TMS influence tinnitus transiently via a direct cortical and indirect somatosensory modulating effect? A combined TMS-tDCS and TENS study. Brain Stimul. 4, 242-252.

Vanneste, S., Plazier, M., Van de Heyning, P., and De Ridder, D. (2011c). Repetitive transcranial magnetic stimulation frequency dependent tinnitus improvement by double cone coil prefrontal stimulation. J. Neurol. Neurosurg. Psychiatr. 82, 1160-1164.

Vanneste, S., Van de Heyning, P. and De Ridder, D. (2011d). Contralateral parahippocampal gammaband activity determines noiselike tinnitus laterality: a region of interest analysis. Neuroscience 199, 481-490.

Vanneste, S., Van de Heyning, P., and De Ridder, D. (2011e). The neural network of phantom sound changes over time: a comparison between recent-onset and chronic tinnitus patients. Eur. J. Neurosci. 34, 718-731.

Vanneste, S., Plazier, M., Ost, J., van der Loo, E., Van de Heyning, P., and De Ridder, D. (2010a). Bilateral dorsolateral prefrontal cortex modulation for tinnitus by transcranial direct current stimulation: a preliminary clinical study. Exp. Brain Res. 202, 779-785.

Vanneste, S., Plazier, M., van der Loo, E., Van de Heyning, P., Congedo, M., and De Ridder, D. (2010b). The neural correlates of tinnitusrelated distress. Neuroimage 52, 470-480.

Voisin, J., Bidet-Caulet, A., Bertrand, O., and Fonlupt, P. (2006). Listening in silence activates auditory areas: a functional magnetic resonance imaging study. J. Neurosci. 26, 273-278.

Wang, H., Tian, J., Yin, D., Jiang, S., Yang, W., Han, D., Yao, S., and Shao M. (2001). Regional glucose metabolic increases in left auditory cortex in tinnitus patients: a preliminary study with positron emission tomography. Chin. Med. J. (Engl) $114,848-851$

Wang, H., Wang, X., and Scheich, H. (1996). LTD and LTP induced by transcranial magnetic stimulation in auditory cortex. Neuroreport 7, 521-525. 
Weisz, N., Dohrmann, K., and Elbert, T. (2007). The relevance of spontaneous activity for the coding of the tinnitus sensation. Prog. Brain Res. 166, 61-70.

Weisz, N., Moratti, S., Meinzer, M., Dohrmann, K., and Elbert, T. (2005a). Tinnitus perception and distress is related to abnormal spontaneous brain activity as measured by magnetoencephalography. PLoS Med.2, e153. doi:10.1371/journal.pmed.0020153

Weisz, N., Wienbruch, C., Dohrmann, K., and Elbert, T. (2005b). Neuromagnetic indicators of auditory cortical reorganization of tinnitus. Brain 128( $\mathrm{Pt} 11)$, 2722-2731.

Yang, S., Weiner, B. D., Zhang, L. S., Cho, S. J., and Bao, S. (2011). Homeostatic plasticity drives tinnitus perception in an animal model. Proc. Natl. Acad. Sci. U.S.A. 108, 14974-14979.

Zhang, J., Zhang, Y., and Zhang, X. (2011). Auditory cortex electrical stimulation suppresses tinnitus in rats. J. Assoc. Res. Otolaryngol. 12, 185-201.

Zunhammer, M., Busch, V., Griesbach, F., Landgrebe, M., Hajak, G., and Langguth, B. (2011). rTMS over the cerebellum modulates temperature detection and pain thresholds through peripheral mechanisms. Brain Stimul. 4, 210-217.

Conflict of Interest Statement: The authors declare that the research was conducted in the absence of any commercial or financial relationships that could be construed as a potential conflict of interest.

Received: 20 January 2012; paperpending published: 13 February 2012; accepted: 01 March 2012; published online: 09 April 2012.

Citation: Langguth B, Schecklmann $M$, Lehner A, Landgrebe M, Poeppl TB,
Kreuzer PM, Schlee W, Weisz N, Vanneste S and De Ridder D (2012) Neuroimaging and neuromodulation: complementary approaches for identifying the neuronal correlates of tinnitus. Front. Syst. Neurosci. 6:15. doi: 10.3389/fnsys.2012.00015

Copyright (C) 2012 Langguth, Schecklmann, Lehner, Landgrebe, Poeppl, Kreuzer, Schlee, Weisz, Vanneste and De Ridder. This is an open-access article distributed under the terms of the Creative Commons Attribution Non Commercial License, which permits noncommercial use, distribution, and reproduction in other forums, provided the original authors and source are credited. 